\title{
The Quantum Inverse Scattering Method Approach to Correlation Functions
}

\author{
A. G. Izergin and V. E. Korepin \\ Leningrad Department of V. A. Steklov Mathematical Institute, Fontanka 27, SU-191011, Leningrad, \\ USSR and Leningrad University, SU-199164, Leningrad, USSR
}

\begin{abstract}
The inverse scattering method approach is developed for calculation of correlation functions in completely integrable quantum models with the $R$ matrix of XXX-type. These models include the one-dimensional Bose-gas and the Heisenberg XXX-model. The algebraic questions of the problem are considered.
\end{abstract}

\section{Introduction}

The quantum inverse scattering method (QISM) [1] is extremely useful for analysis of completely integrable systems. In this paper we formulate the problem of calculation of correlation functions for these models in the frame of QISM. Our approach is essentially different from the one based on the quantum GelfandLevitan equation $[2,3]$. We use results of papers $[4,5]$ where the generalized integrable model was introduced. This model depends on an arbitrary functional parameter. Concrete models such as one-dimensional Bose-gas and the Heisenberg $\mathrm{XXX}$-model can be obtained as special cases at particular values of this parameter. The crucial point is a simple dependence of the generalized model on this functional parameter. We call this generalized model the "one-site" model. By means of this model the simple formula for norms of Bethe wave functions was proved.

In this paper we introduce the "two-site" generalized model which permits us to give a natural formulation of a problem of calculation of correlation functions. This approach can be applied to any model with the $R$-matrix of XXX or XXZ models. Here we restrict ourselves to the XXX-case only.

We deal in this paper with algebraic aspects of the problem, but to clarify the statement of the problem turn now to the one-dimensional Bose-gas with repulsion which is described by the quantum nonlinear Schrödinger equation (so we call this 
model the NS-model). The Hamiltonian of this model is equal to

$$
\mathbf{H}=\int_{0}^{L} d x\left(\partial_{x} \psi^{+} \partial_{x} \psi+c \boldsymbol{\psi}^{+} \boldsymbol{\psi}^{+} \boldsymbol{\psi} \psi\right)-h \int_{0}^{L} d x \boldsymbol{\psi}^{+} \boldsymbol{\psi}
$$

Here $c>0$ is a coupling constant, $h>0-$ a chemical potential, $L-$ a length of a box, $\psi(x)$ - a canonical Bose-field: $\left[\psi(x), \psi^{+}(y)\right]=\delta(x-y), \quad[\psi(x), \psi(y)]=$ $\left[\psi^{+}(x), \psi^{+}(y)\right]=0$. The "bare" vacuum $|0\rangle$ is defined by $\boldsymbol{\psi}(x)|0\rangle=0$, $\langle 0 \mid 0\rangle=1$. Eigenstates of $H$ were constructed in [6-8] by means of the coordinate Bethe's Ansatz:

$$
\left|\Psi_{N}\left(\lambda_{1} \ldots \lambda_{N}\right)\right\rangle=\frac{1}{\sqrt{N !}} \int_{0}^{L} d^{N} z \chi_{N}\left(z_{1} \ldots z_{N} \mid \lambda_{1} \ldots \lambda_{N}\right) \psi^{+}\left(z_{1}\right) \ldots \psi^{+}\left(z_{N}\right)|0\rangle,
$$

where eigenfunction $\chi$ is

$$
\begin{aligned}
& \chi_{N}\left(z_{1} \ldots z_{N}\left|\lambda_{1} \ldots \lambda_{N}\right\rangle=\left\{N ! \prod_{j>k}\left(\lambda_{j k}^{2}+c^{2}\right)\right\}^{-1 / 2}\right. \\
& \quad \times \sum_{P}(-1)^{[P]} \prod_{j>k}\left(\lambda_{P_{j} P_{k}}-i c \varepsilon\left(z_{j}-z_{k}\right)\right) \exp \left\{i \sum_{n=1}^{N} z_{n} \lambda_{P_{n}}\right\} .
\end{aligned}
$$

Here $\lambda_{j k}=\lambda_{j}-\lambda_{k}$; the sum is taken over all the permutations $P$ of $1,2, \ldots N$; $[P]$ denotes a parity of $P$. All the momenta $\lambda_{j}$ are real, different [9] and satisfy the system of "transcendental" equations (s.t.e.) which expresses the periodicity of wave functions:

$$
\exp \left\{i \lambda_{j} L\right\}=\prod_{\substack{k=1 \\ k \neq j}}^{N}\left[\left(\lambda_{j k}+i c\right) /\left(\lambda_{j k}-i c\right)\right] ; j=1, \ldots, N
$$

This system can also be rewritten in the form $\varphi_{j}=0(\bmod 2 \pi)$, where

$$
\varphi_{j}=\lambda_{j} L+\sum_{\substack{k=1 \\ k \neq j}}^{N} \Phi\left(\lambda_{j k}\right)
$$

Here $\Phi(\lambda)=i \ln [(\lambda+i c) /(\lambda-i c)]$ is a scattering phase of bare particles. The eigenvalue of $H$ for wavefunction (1.3) is $\sum_{j=1}^{N}\left(\lambda_{j}^{2}-h\right)$. The norm of the wave function is equal to $[4,10]$

$$
\left\langle\Psi_{N}\left(\lambda_{1} \ldots \lambda_{N}\right) \mid \Psi_{N}\left(\lambda_{1} \ldots \lambda_{N}\right)\right\rangle=\int_{0}^{L} d^{N} z\left|\chi_{N}\right|^{2}=\operatorname{det}_{N}\left(\varphi^{\prime}\right) .
$$

Here the $N \times N$-matrix $\varphi^{\prime}$ is defined as $\varphi_{j k}^{\prime}=\partial \varphi_{j} / \partial \lambda_{k}$.

Consider the current operator $j(x)=\psi^{+}(x) \psi(x)$. We want to study the $N$ particle mean value $\left\langle\Psi_{N}\left|j\left(x_{1}\right) j\left(x_{2}\right)\right| \Psi_{N}\right\rangle$, which is a real positive function of $\mid x_{1}$ $-x_{2} \mid$. For $\left|\Psi_{N}\right\rangle=|\Omega\rangle$ it is a correlation function. The physical vacuum $|\Omega\rangle$ is the 
state with the minimal energy; its construction in the thermodynamical limit ( $N$ $\rightarrow \infty ; L \rightarrow \infty ; N / L=$ Const) see, for example, in [9]. The operator of number of particles for the interval $\left[x_{1}, x_{2}\right]\left(L \geqq x_{2}>x_{1} \geqq 0\right)$ is defined as $\mathbf{Q}_{x_{2} x_{1}}=\int_{x_{1}}^{x_{2}} j(z) d z$. It is easy to see that

$$
\left\langle\Psi_{N}\left|\dot{j}\left(x_{1}\right) \dot{j}\left(x_{2}\right)\right| \Psi_{N}\right\rangle=-\frac{1}{2} \frac{\partial^{2}}{\partial x_{1} \partial x_{2}}\left\langle\Psi_{N}\left|\mathbf{Q}_{x_{2} x_{1}}^{2}\right| \Psi_{N}\right\rangle .
$$

Due to the translation invariance, one can put $\bar{x}_{1}=0$ :

$$
\left\langle\Psi_{N}|j(x) j(0)| \Psi_{N}\right\rangle=\frac{1}{2} \frac{\partial^{2}}{\partial x^{2}}\left\langle\Psi_{N}\left|\mathbf{Q}_{1}^{2}\right| \Psi_{N}\right\rangle,
$$

where

$$
\mathbf{Q}_{1} \equiv \mathbf{Q}_{x 0}=\int_{0}^{x} j(z) d z, \quad x>0 .
$$

So the calculation of $\left\langle\Psi_{N}|j(x) j(0)| \Psi_{N}\right\rangle$ is reduced to the calculation of $\left\langle\Psi_{N}\left|\mathbf{Q}_{1}^{2}\right| \Psi_{N}\right\rangle$. In terms of Bethe's wave functions (1.3), $\left\langle\Psi_{N}\left|\mathbf{Q}_{1}^{2}\right| \Psi_{N}\right\rangle$ is expressed as

$$
\begin{aligned}
\left\langle\Psi_{N}\left|\mathbf{Q}_{1}^{2}\right| \Psi_{N}\right\rangle= & N(N-1) \int_{0}^{x} d^{2} y \int_{0}^{L} d^{N-2} z\left|\chi_{N}\left(y_{1} y_{2} z_{3} \ldots z_{N}\right)\right|^{2} \\
& +\left\langle\Psi_{N}\left|\mathbf{Q}_{1}\right| \Psi_{N}\right\rangle .
\end{aligned}
$$

Here the mean value of operator $\mathbf{Q}_{1}$ is equal to

$$
\left\langle\Psi_{N}\left|\mathbf{Q}_{1}\right| \Psi_{N}\right\rangle=\left\langle\Psi_{N} \mid \Psi_{N}\right\rangle \frac{x N}{L}=\frac{x N}{L} \operatorname{det}_{N}\left(\varphi^{\prime}\right) .
$$

The mean value $\left\langle\Psi_{N}\left|\mathbf{Q}_{1}^{2}\right| \Psi_{N}\right\rangle$ for small $N$ is also easy to calculate: $\left\langle\Psi_{0}\left|\mathbf{Q}_{1}^{2}\right| \Psi_{0}\right\rangle$ $=0 ;\left\langle\Psi_{1}\left|\mathbf{Q}_{1}^{2}\right| \Psi_{1}\right\rangle=x$;

$$
\begin{aligned}
& \left\langle\Psi_{2}\left|\mathbf{Q}_{1}^{2}\right| \Psi_{2}\right\rangle=4 x^{2}+2 x y+\frac{16 c}{\lambda_{12}^{2}+c^{2}} x \\
& +\frac{2}{\lambda_{12}^{2}}\left(\frac{\lambda_{12}+i c}{\lambda_{12}-i c}\right)\left(1-e^{-i x \lambda_{12}}\right)+\frac{2}{\lambda_{12}^{2}}\left(\frac{\lambda_{21}+i c}{\lambda_{21}-i c}\right)\left(1-e^{-i x \lambda_{21}}\right) .
\end{aligned}
$$

Here we denote $y \equiv L-x$. One can see that $\left\langle\Psi_{2}\left|\mathbf{Q}_{1}^{2}\right| \Psi_{2}\right\rangle$ depends on the distance in two essentially different ways: in a polynomial way and in an exponential one. The "exponential" part we call the irreducible part $I_{2}\left(I_{0}=I_{1} \equiv 0\right)$ :

$$
I_{2}=\frac{2}{\lambda_{12}^{2}}\left(\frac{\lambda_{12}+i c}{\lambda_{12}-i c}\right)\left(1-e^{-i x \lambda_{12}}\right)+\frac{2}{\lambda_{12}^{2}}\left(\frac{\lambda_{21}+i c}{\lambda_{21}-i c}\right)\left(1-e^{-i x \lambda_{21}}\right) .
$$

The notion of irreducible part will be extremely useful. Let us give the corresponding definition for arbitrary $N$. Below we'll show that $\left\langle\Psi_{N}\left|\mathbf{Q}_{1}^{2}\right| \Psi_{N}\right\rangle$ can 
be uniquely represented in the form

$$
\left\langle\Psi_{N}\left|\mathbf{Q}_{1}^{2}\right| \Psi_{N}\right\rangle=\sum_{n=0}^{N} \sum_{m=0}^{N-1} J_{m, n}^{(N)} x^{n} y^{m}
$$

Coefficients $J_{m, n}^{(N)}$ here are rational functions of $\lambda_{j}$ and of $\exp \left\{i x \lambda_{j}\right\}(j=1, \ldots, N)$. The $N$-particle irreducible part $I_{N}$ is defined as follows:

$$
I_{N} \equiv J_{00}^{(N)} \text {. }
$$

The irreducible part depends on $x$ through $\exp \left\{ \pm i x \lambda_{j}\right\}$ only, and this dependence can be separated in the following form

$$
I_{N}=\sum_{\{\lambda\}=\left\{\lambda^{+}\right\} \cup\left\{\lambda^{-}\right\} \cup\left\{\lambda^{0}\right\}} e^{-i x \sum_{1}^{n}\left(\lambda_{j}^{+}-\lambda_{j}^{-}\right)} \mathscr{A}_{N}^{n}\left(\left\{\lambda^{+}\right\},\left\{\lambda^{-}\right\},\left\{\lambda^{0}\right\}\right) .
$$

The sum here is taken over all the partitions of the set $\left\{\lambda_{j}\right\}, j=1, \ldots, N$ into three disjoint subsets $\left\{\lambda^{+}\right\},\left\{\lambda^{-}\right\},\left\{\lambda^{0}\right\}$, the number of elements in the subsets being card $\left\{\lambda^{+}\right\}=$card $\left\{\lambda^{-}\right\}=n$; card $\left\{\lambda^{0}\right\}=N-2 n(n \leqq[N / 2])$. The coefficients $\mathscr{A}_{N}^{n}$ are rational functions of momenta $\lambda_{j}$ and do not depend on $x$. They will be called the Fourier coefficients of irreducible part $I_{N}$. We shall see that all the coefficients $J_{m, n}^{(N)}$ in (1.14) can be expressed in terms of irreducible parts $I_{k}, 2 \leqq k \leqq N$. That is the reason why irreducible parts are important.

In this paper we prove also the following properties of irreducible parts. $I_{N}$ is a symmetric function of all the $\lambda_{j}(j=1, \ldots, N)$. It is real and bounded when $\lambda_{j}$ are real. It is a function of the coupling constant $c$ and is small at $c \rightarrow 0$ or $c \rightarrow \infty$ :

$$
I_{N} \sim c^{N-2} \quad \text { at } \quad c \rightarrow 0 ; \quad I_{N} \sim c^{2-N} \quad \text { at } \quad c \rightarrow \infty(N \geqq 2) .
$$

If $x=0$, then $I_{N}=0$. Fourier coefficients have similar properties:

$$
\mathscr{A}_{N}^{k} \sim c^{N-2} \text { at } c \rightarrow 0 ; \mathscr{A}_{N}^{k} \sim c^{2-N} \text { at } c \rightarrow \infty,
$$

and if all the $\lambda_{j}$ are real,

$$
\mathscr{A}_{N}^{k^{*}}\left(\left\{\lambda^{+}\right\},\left\{\lambda^{-}\right\},\left\{\lambda^{0}\right\}\right)=\mathscr{A}_{N}^{k}\left(\left\{\lambda^{-}\right\},\left\{\lambda^{+}\right\},\left\{\lambda^{0}\right\}\right) .
$$

The Fourier coefficients are symmetric functions in all $\lambda_{j}^{+}, \lambda_{j}^{-}$and $\lambda_{j}^{0}$ (separately).

By means of (1.10), (1.14), (1.15) it is easy to calculate $I_{N}$ for small $N$ (see, for example, $I_{3}$ in (8.13), (8.14)), but it is impossible to study the general properties of $I_{N}$. To prove all the properties of $I_{N}$ presented here we introduce the two-site generalized model in Sect. 2. The important formulae concerning "scalar products" in the generalized model are given in Sect. 3. Matrix elements of operators $\exp \left\{\alpha \mathbf{Q}_{1}\right\}$ and $\mathbf{Q}_{1}$ are studied in Sects. 4-6. The definition of irreducible parts and method of their calculation in terms of these quantities are given in Sect. 7. Main results concerning the mean value $\left\langle\Psi_{N}\left|\mathbf{Q}_{1}^{2}\right| \Psi_{N}\right\rangle$ are given in Sect. 8.

The properties of irreducible parts established here will be used by one of us (V.E.K.) in the next publication [11] for calculation of the correlation function of currents in the NS-model. The answer appears to be a series, the $n^{\text {th }}$ term of the series being generated by irreducible part $I_{n}$. 


\section{QISM and the Two-site Generalized Model}

Here we introduce the two-site generalized model, the NS-model (as well as other models with the same $R$-matrix) being the particular case of it. Properties of correlation functions are easy to investigate in the frame of this model.

The main object in QISM (see, for example, [1]) is the monodromy matrix $T(\lambda)$ of the auxiliary linear problem. In our case it is a $2 \times 2$ matrix depending on complex spectral parameter $\lambda$ :

$$
T(\lambda)=\left(\begin{array}{ll}
A(\lambda) ; & B(\lambda) \\
C(\lambda) ; & D(\lambda)
\end{array}\right) .
$$

The matrix elements of $T(\lambda)$ do not commute - they are "quantum operators." Their commutation relations are given by

$$
R(\lambda, \mu) T(\lambda) \otimes T(\mu)=T(\mu) \otimes T(\lambda) R(\lambda, \mu),
$$

where $R(\lambda, \mu)$ is the $\mathrm{XXX}$-model $R$-matrix:

$$
\begin{aligned}
& R(\lambda, \mu)=\left(\begin{array}{cccc}
f(\mu, \lambda) & 0 & 0 & 0 \\
0 & g(\mu, \lambda) & 1 & 0 \\
0 & 1 & g(\mu, \lambda) & 0 \\
0 & 0 & 0 & f(\lambda, \mu)
\end{array}\right) ; \\
& f(\lambda, \mu)=\frac{\lambda-\mu+i c}{\lambda-\mu} ; \quad g(\lambda, \mu)=\frac{i c}{\lambda-\mu} .
\end{aligned}
$$

Let us introduce the two-site generalized model. It is a model with a monodromy matrix $T(\lambda)$ which is a matrix product of two monodromy matrices

$$
\begin{aligned}
T(\lambda) & =T_{2}(\lambda) T_{1}(\lambda) ; \\
T_{i}(\lambda) & =\left(\begin{array}{ll}
A_{i}(\lambda) ; & B_{i}(\lambda) \\
C_{i}(\lambda) ; & D_{i}(\lambda)
\end{array}\right), \quad i=1,2 .
\end{aligned}
$$

The matrix $T_{1}(\lambda)$ can be associated with the first site and $T_{2}(\lambda)$ with the second site of a lattice with two sites. Matrix elements of $T_{i}(\lambda)$ are quantum operators which commute at different sites of the lattice. Operators at the same site commute according to the rule (2.2). The monodromy matrix $T_{i}(\lambda)(i=1,2)$ has the vacuum $|0\rangle_{i}$ - the state in quantum space with the following properties:

$$
\begin{aligned}
& C_{i}(\lambda)|0\rangle_{i}=0 ; \quad A_{i}(\lambda)|0\rangle_{i}=a_{i}(\lambda)|0\rangle_{i} ; \\
& D_{i}(\lambda)|0\rangle_{i}=d_{i}(\lambda)|0\rangle_{i} B_{i}(\lambda)|0\rangle_{i} \neq 0 .
\end{aligned}
$$

The state $|0\rangle=|0\rangle_{2} \otimes|0\rangle_{1}$ is the vacuum for $T(\lambda)(2.5)$ :

$$
C(\lambda)|0\rangle=0 ; \quad A(\lambda)|0\rangle=a(\lambda)|0\rangle ; \quad D(\lambda)|0\rangle=d(\lambda)|0\rangle,
$$

where

$$
a(\lambda)=a_{1}(\lambda) a_{2}(\lambda) ; \quad d(\lambda)=d_{1}(\lambda) d_{2}(\lambda) .
$$


Here $a_{i}(\lambda), d_{i}(\lambda)$ are $c$-number functions which are defined by the choice of concrete models. The crucial point is that there exist monodromy matrices $T_{i}(\lambda)$ for arbitrary functions $a_{i}(\lambda), d_{i}(\lambda)$ [5]. It should be noted that in the XXX-case all such monodromy matrices can be generated by the $L$-operator of the lattice nonlinear Schrödinger model $[12,13]$. It is convenient to use the following notations:

$$
\begin{aligned}
& \ell(\lambda)=a_{1}(\lambda) / d_{1}(\lambda) ; \quad m(\lambda)=a_{2}(\lambda) / d_{2}(\lambda) ; \\
& r(\lambda)=a(\lambda) / d(\lambda)=\ell(\lambda) m(\lambda) .
\end{aligned}
$$

Different functions $\ell(\lambda)$ and $m(\lambda)$ correspond to essentially different models. Function $\ell(\lambda)$ will be the main free functional parameter in the two-site model. It occurs that the dependence of correlation functions on $\ell(\lambda)$ is rather simple and can be explicitly evaluated.

The trace of the monodromy matrix $\tau(\lambda)=A(\lambda)+D(\lambda)$ generates the Hamiltonians of completely integrable systems. Eigenfunctions of $\tau(\lambda)$ are of the form

$$
\left|\psi_{N}\left(\lambda_{1} \ldots \lambda_{N}\right)\right\rangle=\prod_{j=1}^{N} \mathbb{B}\left(\lambda_{j}\right)|0\rangle,
$$

where

$$
\mathbb{B}(\lambda) \equiv B(\lambda) / d(\lambda)
$$

Here all the $\lambda_{j}$ are different [14] and satisfy the system of transcendental equations (s.t.e.)

$$
r_{j} \prod_{\substack{k=1 \\ k \neq j}}^{N}\left(f_{j k} / f_{k j}\right)=1 ; j=1, \ldots, N
$$

Here $f_{j k} \equiv f\left(\lambda_{j}, \lambda_{k}\right)$ and $r_{j} \equiv r\left(\lambda_{j}\right)$. The s.t.e. may be put into the form $\varphi_{j}=0(\bmod 2 \pi)$, where

$$
\varphi_{j}=i \ln r_{j}+i \sum_{\substack{k=1 \\ k \neq j}}^{N} \ln \left(f_{j k} / f_{k j}\right) .
$$

The corresponding eigenvalue of $\tau(\lambda)$ is

$$
\begin{aligned}
\tau(\lambda)\left|\psi_{N}\left(\lambda_{1} \ldots \lambda_{N}\right)\right\rangle & =t_{N}\left(\lambda ; \lambda_{1} \ldots \lambda_{N}\right)\left|\psi_{N}\left(\lambda_{1} \ldots \lambda_{N}\right)\right\rangle \\
t_{N} & =a(\lambda) \prod_{j=1}^{N} f\left(\lambda, \lambda_{j}\right)+d(\lambda) \prod_{j=1}^{N} f\left(\lambda_{j}, \lambda\right) .
\end{aligned}
$$

The dual vacuum $\langle 0|={ }_{2}\langle 0| \otimes_{1}\langle 0|$ satisfies relations $\langle 0| B(\lambda)=0 ;\langle 0| A(\lambda)$ $=a(\lambda)\langle 0| ;\langle 0| D(\lambda)=d(\lambda)\langle 0|$. We put also ${ }_{i}\langle 0 \mid 0\rangle_{i}=\langle 0 \mid 0\rangle=1$. The dual state

$$
\left\langle\psi_{N}\left(\lambda_{1} \ldots \lambda_{N}\right)\right|=\langle 0| \prod_{j=1}^{N} \mathbb{C}\left(\lambda_{j}\right) ; \quad \mathbb{C}(\lambda) \equiv C(\lambda) / d(\lambda)
$$

is an eigenstate of $\tau(\lambda),\left\langle\psi_{N}\right| \tau(\lambda)=t_{N}\left\langle\psi_{N}\right|$, with the same eigenvalue (2.14) if the s.t.e. 
(2.12) is valid. The "norm" is equal to [4]:

$$
\left\langle\psi_{N}\left(\lambda_{1} \ldots \lambda_{N}\right) \mid \psi_{N}\left(\lambda_{1} \ldots \lambda_{N}\right)\right\rangle=c^{N}\left(\prod_{j \neq k} f_{j k}\right) \operatorname{det}_{N}\left(\varphi^{\prime}\right),
$$

where the $N \times N$-matrix $\varphi^{\prime}$ is defined as $\varphi_{j k}^{\prime}=\partial \varphi_{j} / \partial \lambda_{k}$. Notice that eigenfunctions corresponding to different sets of $\lambda_{j}$ are orthogonal due to different eigenvalues (2.14).

The operators of number of particles will play an important role. Operator $\mathbf{Q}_{i}$ of number of particles at the $i^{\text {th }}$ site of the lattice $(i=1,2)$ is defined as follows:

$$
\left[\mathbf{Q}_{i}, T_{j}(\lambda)\right]=\frac{1}{2}\left[\sigma_{3}, T_{i}(\lambda)\right] \delta_{i j} ; \quad \mathbf{Q}_{i}|0\rangle=0 .
$$

A quantum commutator is at the left-hand side here and a matrix commutator of $T_{i}(\lambda)$ with the Pauli matrix $\sigma_{3}$ is at the right-hand side. Operator $\mathbf{Q}$ of complete number of particles is $\mathbf{Q}=\mathbf{Q}_{1}+\mathbf{Q}_{2}$. Eigenvectors of operator $\mathbf{Q}_{i}$ are

$$
\begin{aligned}
& \mathbf{Q}_{i} \prod_{k=1}^{n} \mathbb{B}_{i}\left(\lambda_{k}\right)|0\rangle=n \prod_{k=1}^{n} \mathbb{B}_{i}\left(\lambda_{k}\right)|0\rangle \quad(i=1,2) ; \\
& \langle 0| \prod_{k=1}^{n} \mathbb{C}_{i}\left(\lambda_{k}\right) \mathbf{Q}_{i}=n\langle 0| \prod_{k=1}^{n} \mathbb{C}_{i}\left(\lambda_{k}\right) \quad(i=1,2) .
\end{aligned}
$$

Notice that $\lambda_{j}$ here are arbitrary and are not supposed to satisfy s.t.e. (2.12). The definition of arbitrary function of operator $\mathbf{Q}_{i}$ is quite obvious. Operator $\mathbf{Q}_{1}^{2}$ is of special interest for us. It is however more convenient to consider a generating function $\exp \left\{\alpha \mathbf{Q}_{1}\right\}$. As it is shown in Appendix $\mathrm{A}$ its matrix elements can be represented in the form:

$$
\begin{aligned}
\langle 0| & \prod_{j=1}^{N} \mathbb{C}\left(\lambda_{j}^{C}\right) \exp \left\{\alpha \mathbf{Q}_{1}\right\} \prod_{k=1}^{N} \mathbb{B}\left(\lambda_{k}^{B}\right)|0\rangle \\
= & \sum_{\left\{\lambda^{B}\right\}=\left\{\lambda_{I}^{B}\right\} \cup\left\{\lambda_{I I}^{B}\right\}} \sum_{\{\lambda\}=\left\{\lambda_{I}^{C}\right\} \cup\left\{\lambda_{I I}^{C}\right\}} \exp \left\{\alpha n_{1}\right\} \\
& \times\left\langle 0\left|\prod_{I} \mathbb{C}_{1}\left(\lambda_{I}^{C}\right) \prod_{I} \mathbb{B}_{1}\left(\lambda_{I}^{B}\right)\right| 0\right\rangle\left\langle 0\left|\prod_{I I} \mathbb{C}_{2}\left(\lambda_{I I}^{C}\right) \prod_{I I} \mathbb{B}_{2}\left(\lambda_{I I}^{B}\right)\right| 0\right\rangle \\
& \times\left(\prod_{I} m\left(\lambda_{I}^{B}\right)\right)\left(\prod_{I I} \ell\left(\lambda_{I I}^{C}\right)\right)\left(\prod_{I, I I} f\left(\lambda_{I}^{B}, \lambda_{I I}^{B}\right)\right)\left(\prod_{I, I I} f\left(\lambda_{I I}^{C}, \lambda_{I}^{C}\right)\right) .
\end{aligned}
$$

Here the sum is taken over all the partitions of the set $\left\{\lambda_{j}^{B} ; j=1, \ldots, N\right\}$ into two disjoint subsets $\left\{\lambda_{I}^{B}\right\}$ and $\left\{\lambda_{I I}^{B}\right\}$, and over similar partitions of the set $\left\{\lambda^{C}\right\}$. These partitions are independent except that $\operatorname{card}\left\{\lambda_{I}^{B}\right\}=\operatorname{card}\left\{\lambda_{I}^{C}\right\}=n_{1} ; \operatorname{card}\left\{\lambda_{I I}^{B}\right\}=$ $\operatorname{card}\left\{\lambda_{I I}^{C}\right\}=n_{2}=N-n_{1}$. Product $\prod_{I}$ denotes the product over all the $\lambda \in\left\{\lambda_{I}\right\}$, and thus contains $n_{1}$ factors. Product $\prod_{I, I I}^{I}$ denotes double product over all $\lambda \in\left\{\lambda_{I}\right\}$ and over all $\lambda \in\left\{\lambda_{I I}\right\}$ and contains $n_{1} n_{2}$ factors. Notice that values of $\lambda^{C}, \lambda^{B}$ in (2.19) are quite arbitrary. This equation is a basic one for investigation in the two-site model 
the mean value

$$
\left\langle\psi_{N}\left|\mathbf{Q}_{1}^{2}\right| \psi_{N}\right\rangle=\left\langle\psi_{N}\left(\lambda_{1} \ldots \lambda_{N}\right)\left|\mathbf{Q}_{1}^{2}\right| \psi_{N}\left(\lambda_{1} \ldots \lambda_{N}\right)\right\rangle,
$$

in terms of which correlation functions can be expressed (as it will be seen later).

Let us discuss the connection of the two-site model with the NS-model. The NSmodel was imbedded in QISM in papers $[1,15,16]$. The monodromy matrix $T_{\mathrm{NS}}(\lambda)$ of this model is constructed in a standard way by means of local $L$-operators. Vacuum eigenvalues of $A(\lambda)$ and $D(\lambda)$ are

$$
\begin{aligned}
& a_{\mathrm{NS}}(\lambda)=\exp \{-i \lambda L / 2\} ; \quad d_{\mathrm{NS}}(\lambda)=\exp \{i \lambda L / 2\} ; \\
& r_{\mathrm{NS}}(\lambda)=\exp \{-i \lambda L\} .
\end{aligned}
$$

The Hamiltonian (1.1) can be expressed in terms of $\tau(\lambda)$ by means of trace identities [17]. Equation (2.5) has the following meaning in the NS-model: $T_{1}(\lambda)$ is the monodromy matrix of the NS-model for the interval $[0, x], T_{2}(\lambda)$ is the monodromy matrix for the interval $[x, L]$. So we have the correspondence:

$$
\ell_{\mathrm{NS}}(\lambda)=\exp \{-i \lambda x\} ; m_{\mathrm{NS}}(\lambda)=\exp \{i \lambda(x-L)\} .
$$

Operator $\mathbf{Q}_{1}$ introduced in the two-site model by Eq. (2.17) turns into operator $\mathbf{Q}_{1}$ (1.9). So the calculation of the mean value(1.7) in the NS-model which is necessary to calculate the correlation function is reduced to the calculation of the mean value (2.20) in the generalized model. This reduction is very useful due to the arbitrary functional parameter $\ell(\lambda)(2.9)$ existing in the generalized model.

Below we'll study the mean value (2.20) in the two-site model. By means of Eq. (2.19) this object is expressed in terms of "scalar products" $\left\langle 0\left|\prod \mathbb{C}(\lambda) \prod \mathbb{B}(\lambda)\right| 0\right\rangle$. The next section is devoted to the description of properties of these scalar products.

\section{Scalar Products}

We call "scalar product" a quantity

$$
\left\langle 0\left|\prod_{j=1}^{N} \mathbb{C}\left(\lambda_{j}^{C}\right) \prod_{k=1}^{N} \mathbb{B}\left(\lambda_{k}^{B}\right)\right| 0\right\rangle
$$

which is a symmetric function of all $\lambda_{j}^{C}$ and a symmetric function of all $\lambda_{k}^{B}$. Here all $2 N$ momenta $\lambda^{C}, \lambda^{B}$ are different and arbitrary (the s.t.e. (2.12) is not in general supposed to be fulfilled). Note that the number of operators $\mathbb{B}$ in (3.1) is equal to the number of operators $\mathbb{C}$; otherwise the scalar product is equal to zero. Scalar products can be calculated by means of the commutation relations (2.2). For instance, $\left\langle 0\left|\mathbb{C}\left(\lambda^{C}\right) \mathbb{B}\left(\lambda^{B}\right)\right| 0\right\rangle=g\left(\lambda^{C}, \lambda^{B}\right)\left[r\left(\lambda^{C}\right)-r\left(\lambda^{B}\right)\right]$. For $N$ arbitrary the dependence of scalar products on vacuum eigenvalues $r(\lambda)$ can be explicitly extracted [4]:

$$
\left\langle 0\left|\prod_{j=1}^{N} \mathbb{C}\left(\lambda_{j}^{C}\right) \prod_{k=1}^{N} \mathbb{B}\left(\lambda_{k}^{B}\right)\right| 0\right\rangle=\sum_{\text {part }}\left(\prod_{j=1}^{N} r\left(\lambda_{j}^{(p r)}\right)\right) \mathbb{K}_{N}(\text { part })
$$

The sum here is taken over all the partitions of the set $\left\{\lambda^{C}\right\}_{N} \cup\left\{\lambda^{B}\right\}_{N}$ into two disjoint subsets $\left\{\lambda^{(p r)}\right\}_{N}$ and $\left\{\lambda^{(a b)}\right\}_{N}$ (subindex $N$ in $\{\lambda\}_{N}$ means the number of elements in this set). Coefficients $\mathbb{K}_{N}$ do not depend on $r(\lambda)$ being rational functions of $2 N$ variables $\lambda^{B}, \lambda^{C}$ decreasing in each $\lambda$ as $1 / \lambda$ at $\lambda \rightarrow \infty$ and other $\lambda^{\prime}$ s fixed. 
Return now to the scalar product (3.2). It depends on values of an arbitrary function $r(\lambda)$ at $2 N$ points $\lambda_{j}^{C}, \lambda_{k}^{B}$. Due to the arbitrariness of function $r(\lambda)$ the values $r\left(\lambda^{B, C}\right)$ can be considered as $2 N$ independent complex variables:

$$
r_{j}^{C} \equiv r\left(\lambda_{j}^{C}\right) ; \quad r_{k}^{B} \equiv r\left(\lambda_{k}^{B}\right) \quad(j, k=1, \ldots, N) .
$$

So scalar product (3.2) is a function of $4 N$ independent variables: $\left\{\lambda^{C}\right\}_{N},\left\{\lambda^{B}\right\}_{N},\left\{r^{C}\right\}_{N}$, $\left\{r^{B}\right\}_{N}$.

Properties of scalar products can be restored from paper [4]. The most important property is that scalar product (3.2) has a simple pole when $\lambda_{j}^{C} \rightarrow$ $\lambda_{k}^{B}(j, k=1, \ldots, N)$, the residue being also some scalar product. For example, at $\lambda_{N}^{C} \rightarrow \lambda_{N}^{B} \rightarrow \lambda_{N}$ one has (the general case is obvious due to the symmetry):

$$
\begin{gathered}
\left.\left\langle 0\left|\prod_{j=1}^{N} \mathbb{C}\left(\lambda_{j}^{C}\right) \prod_{k=1}^{N} \mathbb{B}\left(\lambda_{k}^{B}\right)\right| 0\right\rangle\right|_{\lambda_{N}^{C} \rightarrow \lambda_{N}^{B}} \\
\rightarrow \frac{i c}{\lambda_{N}^{C}-\lambda_{N}^{B}}\left(r_{N}^{C}-r_{N}^{B}\right)\left(\prod_{j=1}^{N-1} f_{N j}^{B} f_{N j}^{C}\right)\left\langle 0\left|\prod_{j=1}^{N-1} \mathbb{C}\left(\lambda_{j}^{C}\right) \prod_{k=1}^{N-1} \mathbb{B}\left(\lambda_{k}^{B}\right)\right| 0\right\rangle^{\bmod } \\
f_{N j}^{B} \equiv f\left(\lambda_{N}, \lambda_{j}^{B}\right) ; \quad f_{N j}^{C} \equiv f\left(\lambda_{N}, \lambda_{j}^{C}\right) .
\end{gathered}
$$

The scalar product at the right hand side must be calculated with the modified vacuum values $\tilde{a}(\lambda)=a(\lambda) f\left(\lambda, \lambda_{N}\right) ; \partial(\lambda)=d(\lambda) f\left(\lambda_{N}, \lambda\right)$. Due to this modification one has:

$$
\begin{aligned}
& \left\langle 0\left|\prod_{j=1}^{N-1} \mathbb{C}\left(\lambda_{j}^{C}\right) \prod_{k=1}^{N-1} \mathbb{B}\left(\lambda_{k}^{B}\right)\right| 0\right\rangle^{\text {mod }} \\
& \quad=\sum_{\text {part }}\left(\prod_{j=1}^{N-1} \tilde{r}\left(\lambda_{j}^{(p r)}\right)\right) \mathbb{K}_{N-1}(\text { part }) ; \quad \operatorname{card}\left\{\lambda^{(p r)}\right\}_{N-1}=N-1,
\end{aligned}
$$

where

$$
\tilde{r}(\lambda)=r(\lambda)\left[f\left(\lambda, \lambda_{N}\right) / f\left(\lambda_{N}, \lambda\right)\right]
$$

It is essential that coefficients $\mathbb{K}_{N-1}$ are not modified: they are just the same as in Eq. (3.2) at $N \rightarrow N-1$. Notice that modified scalar product in Eq. (3.4) does not contain $r_{N}^{B, C}$, and $\lambda_{N}$ is included in $\tilde{r}(\lambda)$ (see (3.5), (3.6)).

In physical cases variables $r_{j}$ are the values of smooth function $r(\lambda)$ at different points (see Eq. (3.3)). In this special case the residue in Eq. (3.4) becomes zero; the corresponding limit is finite. At $\lambda_{N}^{C} \rightarrow \lambda_{N}^{B} \rightarrow \lambda_{N}$ the dependence of the scalar product on the vacuum eigenvalue at point $\lambda_{N}$ is represented naturally in terms of two variables: $r_{N}=r\left(\lambda_{N}\right)$ and $z_{N}=i \partial[\ln r(\lambda)] /\left.\partial \lambda\right|_{\lambda=\lambda_{N}}$. The dependence on $z_{N}$ is linear, the coefficient at $z_{N}$ being essentially the residue in Eq. (3.4):

$$
\begin{aligned}
& \frac{\partial}{\partial z_{N}}\left\langle 0\left|\left(\prod_{j=1}^{N-1} \mathbb{C}\left(\lambda_{j}^{C}\right)\right) \mathbb{C}\left(\lambda_{N}\right) \mathbb{B}\left(\lambda_{N}\right)\left(\prod_{k=1}^{N-1} \mathbb{B}\left(\lambda_{k}^{B}\right)\right)\right| 0\right\rangle \\
& \quad=\operatorname{cr}_{N}\left(\prod_{k=1}^{N-1} f_{N k}^{B} f_{N k}^{C}\right)\left\langle 0\left|\prod_{j=1}^{N-1} \mathbb{C}\left(\lambda_{j}^{C}\right) \prod_{k=1}^{N-1} \mathbb{B}\left(\lambda_{k}^{B}\right)\right| 0\right\rangle^{\mathrm{mod}} .
\end{aligned}
$$


Let us consider now the scalar product at the limit $\lambda_{j}^{C} \rightarrow \lambda_{j}^{B} \rightarrow \lambda_{j}(j=1, \ldots, N)$; all the $\lambda_{j}$ are different. In this case the scalar product depends on $3 N$ complex variables $\{\lambda\}_{N},\{z\}_{N},\{r\}_{N}$. Here

$$
z_{j}=i \partial[\ln r(\lambda)] /\left.\partial \lambda\right|_{\lambda=\lambda_{j}} .
$$

Equation (3.7) is valid also in this case; in the scalar product at the right-hand side not only $r(\lambda)$ is modified according to (3.6) but also $z_{j}$ according to the rule:

$$
\tilde{z}_{j}=z_{j}+K_{j N} ; \quad K_{j N}=K\left(\lambda_{j}, \lambda_{N}\right)=\frac{2 c}{\left(\lambda_{j}-\lambda_{N}\right)^{2}+c^{2}} ; j=1, \ldots, N-1 .
$$

Discuss now the case where only the part of $\left\{\lambda^{C}\right\}$ coincides with the part of $\left\{\lambda^{B}\right\}$. Then the scalar product (3.1) depends on $r_{j}$ and $z_{j}$ of coinciding $\lambda$ 's and on $r_{j}^{B, C}$ of remaining $\lambda$ 's. Equation (3.7) is also valid in this case.

Finally consider the situation when $\lambda_{j}^{C}=\lambda_{j}^{B}=\lambda_{j}(j=1, \ldots, N)$, all $\lambda_{j}$ are different and satisfy s.t.e. (2.12). In this case $r_{j}$ are expressed by s.t.e. as explicit rational functions of $\lambda$ 's, the scalar product depends only on $\{\lambda\}_{N}$ and $\{z\}_{N}$ and is called the "norm" of the wave function (the explicit expression is given in (2.16)). Equation (3.7) remains valid. It should be noted that in this case the scalar product at the righthand side of (3.7) can be also considered as the norm corresponding to the modified s.t.e.

$$
\tilde{r}_{j} \prod_{k \neq j}^{N}\left(f_{j k} / f_{k j}\right)=1 ; \quad \tilde{r}_{j}=r_{j}\left(f_{j N} / f_{N j}\right) ; \quad j=1, \ldots, N-1,
$$

which is valid due to (2.12).

\section{Properties of Operator $\exp \left\{\alpha Q_{1}\right\}$ in the Generalized Model}

In the previous section we considered properties of scalar products which are valid also in the two-site model. This model, however, permits us to consider operators $\mathbf{Q}_{i}$ (2.17). Properties of their matrix elements are investigated in the same way as properties of scalar products. Consider the matrix element $\langle 0| \prod \mathbb{C}\left(\lambda^{C}\right) \times$ $\exp \left\{\alpha \mathbf{Q}_{1}\right\} \prod \mathbb{B}\left(\lambda^{B}\right)|0\rangle$ with all $\lambda^{B}$ and $\lambda^{C}$ arbitrary. Equation (2.19) shows that this matrix element depends on $6 N$ complex variables: on $2 N$ momenta $\lambda_{j}^{C}, \lambda_{k}^{B}$ and on $4 N$ variables $\ell_{j}^{C}, \ell_{k}^{B}, m_{j}^{C}, m_{k}^{B}(2.9)$, being a rational function of all these variables. So we denote:

$$
\begin{aligned}
& \left\langle 0\left|\prod_{j=1}^{N} \mathbb{C}\left(\lambda_{j}^{C}\right) \exp \left\{\alpha \mathbf{Q}_{1}\right\} \prod_{k=1}^{N} \mathbb{B}\left(\lambda_{k}^{B}\right)\right| 0\right\rangle \\
& \equiv \mathbb{M}_{N}^{\alpha}\left(\left\{\lambda^{C}\right\}_{N},\left\{\lambda^{B}\right\}_{N},\left\{\ell^{C}\right\}_{N},\left\{\ell^{B}\right\}_{N},\left\{m^{C}\right\}_{N},\left\{m^{B}\right\}_{N}\right) .
\end{aligned}
$$

Due to commutation relations (2.2): $[\mathbb{C}(\lambda), \mathbb{C}(\mu)]=[\mathbb{B}(\lambda), \mathbb{B}(\mu)]=0$. Hence $\mathbb{M}_{N}^{\alpha}$ is a symmetric function with respect to replacement of triples $\left(\lambda_{j}^{C}, \ell_{j}^{C}, m_{j}^{C}\right) \leftrightarrow$ $\left(\lambda_{k}^{C}, \ell_{k}^{C}, m_{k}^{C}\right)$ and with respect to $\left(\lambda_{i}^{B}, \ell_{i}^{B}, m_{i}^{B}\right) \leftrightarrow\left(\lambda_{n}^{B}, \ell_{n}^{B}, m_{n}^{B}\right)$. The main property of $\mathbb{M}_{N}^{\alpha}$ is that it has first-order poles at $\lambda_{j}^{C} \rightarrow \lambda_{k}^{B}$, the residue being expressed in terms of 
$\mathbb{M}_{N-1}^{\alpha}$. One has for (4.1) if $\lambda_{N}^{C} \rightarrow \lambda_{N}^{B} \rightarrow \lambda_{N}$ (other possibilities are easily restored from the symmetry):

$$
\begin{aligned}
& \left.\mathbb{M}_{N}^{\alpha}\right|_{\lambda_{N}^{C} \rightarrow \lambda_{N}^{B}}=\frac{i c}{\lambda_{N}^{C}-\lambda_{N}^{B}}\left[\ell_{N}^{C} m_{N}^{B}-r_{N}^{B}\right] e^{\alpha}\left(\prod_{j=1}^{N-1} f_{N j}^{C} f_{N j}^{B}\right) \\
& \quad \times \mathbb{M}_{N-1}^{\alpha}\left(\left\{\lambda_{j}^{C}\right\}_{N-1},\left\{\lambda_{j}^{B}\right\}_{N-1},\left\{\tilde{\ell}_{j}^{C}\right\}_{N-1},\left\{\tilde{\ell}_{j}^{B}\right\}_{N-1},\left\{m_{j}^{C}\right\}_{N-1},\left\{m_{j}^{B}\right\}_{N-1}\right) \\
& \quad+\frac{i c}{\lambda_{N}^{C}-\lambda_{N}^{B}}\left[r_{N}^{C}-\ell_{N}^{C} m_{N}^{B}\right]\left(\prod_{j=1}^{N-1} f_{N j}^{C} f_{N j}^{B}\right) \\
& \quad \times \mathbb{M}_{N-1}^{\alpha}\left(\left\{\lambda_{j}^{C}\right\}_{N-1},\left\{\lambda_{j}^{B}\right\}_{N-1},\left\{\ell_{j}^{C}\right\}_{N-1},\left\{\ell_{j}^{B}\right\}_{N-1},\left\{\tilde{m}_{j}^{C}\right\}_{N-1},\left\{\tilde{m}_{j}^{B}\right\}_{N-1}\right) .
\end{aligned}
$$

Here $f_{N j}^{C, B} \equiv f\left(\lambda_{N}, \lambda_{j}^{C, B}\right)$ and

$$
\tilde{\ell}_{j}^{C, B}=\ell_{j}^{C, B}\left(f_{j N}^{C, B} / f_{N j}^{C, B}\right) ; \quad \tilde{m}_{j}^{C, B}=m_{j}^{C, B}\left(f_{j N}^{C, B} / f_{N j}^{C, B}\right) .
$$

(Compare with (3.5),(3.6)). Equation (4.2) can be obtained by substitution of Eq. (3.4) into Eq. (2.19). Notice that $\mathbb{M}_{N-1}^{\alpha}$ here are modified according to the rule (4.3) (compare with (3.6).) It is essential that $\mathbb{M}_{N-1}^{\alpha}$ does not depend on $\ell_{N}, m_{N}$. The variable $\lambda_{N}$ enters only into the modified $\tilde{\ell}_{j}$ and $\tilde{m}_{j}$ in $\mathbb{M}_{N-1}^{\alpha}$.

Formula (4.2) is a basic one for the investigation of the matrix element $\mathbb{M}$. It can be considered in two different ways depending on the smoothness of functions $\ell(\lambda)$, $m(\lambda)$.

(1) In physical cases variables $\ell_{j}$ and $m_{j}$ are the values of smooth functions $\ell(\lambda)$ and $m(\lambda)$ :

$$
\ell_{j}^{B, C}=\ell\left(\lambda_{j}^{B, C}\right) ; \quad m_{j}^{B, C}=m\left(\lambda_{j}^{B, C}\right)
$$

(for the NS-model $\ell(\lambda)=\exp \{-i x \lambda\} ; m(\lambda)=\exp \{i(x-L) \lambda\}$ ). In this case the residue in (4.2) becomes zero; the corresponding limit is finite. At $\lambda_{N}^{C} \rightarrow \lambda_{N}^{B} \rightarrow \lambda_{N}$ the dependence of the scalar product on vacuum eigenvalues at point $\lambda_{N}$ is represented in terms of four variables:

$$
\ell_{N}=\ell\left(\lambda_{N}\right) ; \quad m_{N}=m\left(\lambda_{N}\right)
$$

and

$$
x_{N}=i \partial[\ln \ell(\lambda)] /\left.\partial \lambda\right|_{\lambda=\lambda_{N}} ; \quad y_{N}=i \partial[\ln m(\lambda)] /\left.\partial \lambda\right|_{\lambda=\lambda_{N}} .
$$

The dependence on $x_{N}$ and $y_{N}$ is linear, the coefficients at $x_{N}$ and $y_{N}$ being essentially the residue in Eq. (4.2):

$$
\begin{aligned}
& \partial \mathbb{M}_{N}^{\alpha} / \partial x_{N}=\operatorname{cr}\left(\lambda_{N}\right) \exp \{\alpha\}\left(\prod_{j=1}^{N-1} f_{N j}^{C} f_{N j}^{B}\right) \\
& \quad \times \mathbb{M}_{N-1}^{\alpha}\left(\left\{\lambda_{j}^{C}\right\}_{N-1},\left\{\lambda_{j}^{B}\right\}_{N-1},\left\{\tilde{\ell}_{j}^{C}\right\}_{N-1},\left\{\tilde{\ell}_{j}^{B}\right\}_{N-1},\left\{m_{j}^{C}\right\}_{N-1},\left\{m_{j}^{B}\right\}_{N-1}\right) \\
& \partial \mathbb{M}_{N}^{\alpha} / \partial y_{N}=\operatorname{cr}\left(\lambda_{N}\right)\left(\prod_{j=1}^{N-1} f_{N J}^{C} f_{N j}^{B}\right) \\
& \quad \times \mathbb{M}_{N-1}^{\alpha}\left(\left\{\lambda_{j}^{C}\right\}_{N-1},\left\{\lambda_{j}^{B}\right\}_{N-1},\left\{\ell_{j}^{C}\right\}_{N-1},\left\{\ell_{j}^{B}\right\}_{N-1},\left\{\tilde{m}_{j}^{C}\right\}_{N-1},\left\{\tilde{m}_{j}^{B}\right\}_{N-1}\right)
\end{aligned}
$$

The modification here is to be done using the same rule (4.3).

Let us consider now the matrix element at the limit $\lambda_{j}^{C} \rightarrow \lambda_{j}^{B} \rightarrow \lambda_{j}(j=1, \ldots, N)$, all 
the $\lambda_{j}$ being different. In this case $\mathbb{M}_{N}$ depends on $5 N$ complex variables (compare with (3.8)):

$$
\mathbb{M}_{N}^{\alpha} \equiv M_{N}^{\alpha}\left(\{\lambda\}_{N},\{x\}_{N},\{y\}_{N},\{\ell\}_{N},\{m\}_{N}\right)
$$

where

$$
x_{k}=i \partial[\ln \ell(\lambda)] /\left.\partial \lambda\right|_{\lambda=\lambda_{k}} ; \quad y_{k}=i \partial[\ln m(\lambda)] /\left.\partial \lambda\right|_{\lambda=\lambda_{k}} .
$$

Notice that for the NS-model $x_{j}=x ; y_{j}=L-x$. The linearity in each $x_{j}, y_{j}$ is preserved and Eqs. (4.5), (4.6) are valid also for $M_{N}$ (4.7):

$$
\begin{aligned}
\partial M_{N}^{\alpha} / \partial x_{N}= & \operatorname{cr}\left(\lambda_{N}\right) \exp \{\alpha\}\left(\prod_{j=1}^{N-1} f_{N j} f_{N j}\right) \\
& \times M_{N-1}^{\alpha}\left(\left\{\lambda_{j}\right\}_{N-1},\left\{\tilde{x}_{j}\right\}_{N-1},\left\{y_{j}\right\}_{N-1},\left\{\tilde{\ell}_{j}\right\}_{N-1},\left\{m_{j}\right\}_{N-1}\right), \\
\partial M_{N}^{\alpha} / \partial y_{N}= & \operatorname{cr}\left(\lambda_{N}\right)\left(\prod_{j=1}^{N-1} f_{N j} f_{N j}\right) \\
& \times M_{N-1}^{\alpha}\left(\left\{\lambda_{j}\right\}_{N-1},\left\{x_{j}\right\}_{N-1},\left\{\tilde{y}_{j}\right\}_{N-1},\left\{\ell_{j}\right\}_{N-1},\left\{\tilde{m}_{j}\right\}_{N-1}\right) .
\end{aligned}
$$

Here $f_{N j} \equiv f\left(\lambda_{N}, \lambda_{j}\right)$; the modification of $\ell$ and $m$ is done according to (4.3) and

$$
\tilde{x}_{j}=x_{j}+K_{j N} ; \quad \tilde{y}_{j}=y_{j}+K_{j N} \quad(j=1, \ldots, N-1)
$$

with $K_{j N}$ defined in (3.9).

Consider now the case where not only $\lambda_{j}^{B}=\lambda_{j}^{C}=\lambda_{j}(j=1, \ldots, N)$ but also s.t.e. (2.12) for $\lambda_{j}$ is valid. The matrix element in this case is the mean value with respect to eigenfunctions $\psi_{N}(2.10),(2.15)$ :

$$
\begin{aligned}
M_{N}^{\alpha} & =\left\langle\psi_{N}\left(\lambda_{1} \ldots \lambda_{N}\right)\left|\exp \left\{\alpha \mathbf{Q}_{1}\right\}\right| \psi_{N}\left(\lambda_{1} \ldots \lambda_{N}\right)\right\rangle \\
& \equiv \mathscr{M}_{N}^{\alpha}\left(\{\lambda\}_{N},\{x\}_{N},\{y\}_{N},\{\ell\}_{N}\right) .
\end{aligned}
$$

Here we have written down explicitly all $4 N$ independent variables. The matter is that variables $m_{j}$ in this case can be expressed in terms of remaining variables due to s.t.e. (2.12):

$$
m_{j}=\ell_{j}^{-1} \prod_{k \neq j}\left(f_{k j} / f_{j k}\right) .
$$

Equations (4.9), (4.10) are rewritten in the form:

$$
\begin{aligned}
\partial \mathscr{M}_{N}^{\alpha} / \partial x_{N}= & \exp \{\alpha\}\left(\prod_{j=1}^{N-1} f_{N j} f_{j N}\right) \\
& \times \mathscr{M}_{N-1}^{\alpha}\left(\left\{\lambda_{j}\right\}_{N-1},\left\{\tilde{x}_{j}\right\}_{N-1},\left\{y_{j}\right\}_{N-1},\left\{\tilde{\ell}_{j}\right\}_{N-1}\right) \\
\partial \mathscr{M}_{N}^{\alpha} / \partial y_{N}= & c\left(\prod_{j=1}^{N-1} f_{N j} f_{j N}\right) \\
& \times \mathscr{M}_{N-1}^{\alpha}\left(\left\{\lambda_{j}\right\}_{N-1},\left\{x_{j}\right\}_{N-1},\left\{\tilde{y}_{j}\right\}_{N-1},\left\{\ell_{j}\right\}_{N-1}\right)
\end{aligned}
$$

Modification here is made according to rules (4.3), (4.11). Formulae (4.14), (4.15) are very important because they give an opportunity to restore all the coefficients $J_{m, n}$ in 
Eq. (1.14) in terms of irreducible parts (1.15). This restoration is done in [11].

(2) Return now to Eqs. (4.1), (4.2) and consider another situation. Suppose that $\left\{\lambda^{C}\right\}$ as well as $\left\{\lambda^{B}\right\}$ satisfy s.t.e.(2.12) but these sets do not coincide. In this case "bra" and "ket" in (4.1) are different eigenfunctions (2.10), (2.15). The corresponding matrix element we call "form factor":

$$
\begin{aligned}
\mathbb{M}_{N}^{\alpha} & =\left\langle\psi_{N}\left(\lambda_{1}^{C} \ldots \lambda_{N}^{C}\right)\left|\exp \left\{\alpha \mathbf{Q}_{1}\right\}\right| \psi_{N}\left(\lambda_{1}^{B} \ldots \lambda_{N}^{B}\right)\right\rangle \\
& \equiv F_{N}^{\alpha}\left(\left\{\lambda^{C}\right\},\left\{\lambda^{B}\right\},\left\{\ell^{C}\right\},\left\{\ell^{B}\right\}\right) .
\end{aligned}
$$

The variables $m_{j}^{B, C}$ presented in (4.1) are here expressed by means of the s.t.e. It is easy to obtain from formula (4.2) that:

$$
\begin{aligned}
\left.F_{N}^{\alpha}\right|_{\lambda_{N}^{C} \rightarrow \lambda_{N}^{B} \rightarrow \lambda_{N}}= & \frac{i c}{\lambda_{N}^{C}-\lambda_{N}^{B}}\left[\ell{ }_{N}^{C}\left(\ell_{N}^{B}\right)^{-1}-1\right] \exp \{\alpha\} \\
& \times\left(\prod_{j=1}^{N-1} f_{N j}^{C} f_{j N}^{B}\right) F_{N-1}^{\alpha}\left(\left\{\lambda^{C}\right\}_{N-1},\left\{\lambda^{B}\right\}_{N-1},\left\{\tilde{\ell}^{C}\right\}_{N-1},\left\{\tilde{\ell}^{B}\right\}_{N-1}\right) \\
& +\frac{i c}{\lambda_{N}^{C}-\lambda_{N}^{B}}\left[\left(\prod_{j=1}^{N-1} f_{j N}^{C} f_{N j}^{B}\right)-\ell_{N}^{C}\left(\ell_{N}^{B}\right)^{-1}\left(\prod_{j=1}^{N-1} f_{N j}^{C} f_{j N}^{B}\right)\right] \\
& \times F_{N-1}^{\alpha}\left(\left\{\lambda^{C}\right\}_{N-1},\left\{\lambda^{B}\right\}_{N-1},\left\{\ell^{C}\right\}_{N-1},\left\{\ell^{B}\right\}_{N-1}\right) .
\end{aligned}
$$

The modification of $\ell$ here is defined in (4.3). These properties are used in the next Section to obtain a representation for $F_{N}^{\alpha}$ which permits to study irreducible parts.

\section{Representation of the Form Factor of Operator $\exp \left\{\alpha Q_{1}\right\}$}

Here we consider form factor $F_{N}^{\alpha}$ (4.16). Our aim is to obtain a representation for it which is similar to the one for scalar products given in [4].

Discuss at first the dependence of $F_{N}^{\alpha}$ on variables $\ell_{j}^{C, B}$. It is more convenient here to use notations (4.4) denoting $\ell\left(\lambda_{j}^{C, B}\right) \equiv \ell_{j}^{C, B}$ (due to the arbitrariness of function $\ell(\lambda)$, its values $\ell\left(\lambda_{j}^{C, B}\right)$ can be considered as independent variables). The dependence of $F$ on these variables can be explicitly separated as follows:

$$
\begin{aligned}
& F_{N}^{\alpha}\left(\left\{\lambda^{C}\right\}_{N},\left\{\lambda^{B}\right\}_{N},\left\{\ell^{C}\right\}_{N},\left\{\ell^{B}\right\}_{N}\right) \\
& \quad=\sum_{\text {part }}\left(\prod_{p r} \ell\left(\lambda_{p r}^{C}\right)\right)\left(\prod_{p r} \ell^{-1}\left(\lambda_{p r}^{B}\right)\right) \mathbb{R}_{N} \text { (part). }
\end{aligned}
$$

Here the sum is taken over all the partitions of set $\left\{\lambda^{C}\right\}_{N}$ into two disjoint subsets $\left\{\lambda_{p r}^{C}\right\}_{n}$ and $\left\{\lambda_{a b}^{C}\right\}_{N-n}$ and over partitions of set $\left\{\lambda^{B}\right\}_{N}$ into two disjoint subsets $\left\{\lambda_{p r}^{B}\right\}_{n}$ and $\left\{\lambda_{a b}^{B}\right\}_{N-n}$. These partitions are independent except that card $\left\{\lambda_{p r}^{C}\right\}_{n}=\operatorname{card}\left\{\lambda_{p r}^{B}\right\}_{n}$ $=n ; \operatorname{card}\left\{\lambda_{a b}^{C}\right\}_{N-n}=\operatorname{card}\left\{\lambda_{a b}^{B}\right\}_{N-n}=N-n$. Product $\prod_{p r} \ell\left(\lambda_{p r}^{C}\right)$ denotes the product of $n$ factors $\ell\left(\lambda_{j}^{C}\right) ; \lambda_{j}^{C} \in\left\{\lambda_{p r}^{C}\right\}_{n}$. Product $\prod_{p r} \ell^{-1}\left(\lambda_{p r}^{B}\right)$ denotes the product on $n$ factors $\ell^{-1}\left(\lambda_{j}^{B}\right) ; \lambda_{j}^{B} \in\left\{\lambda_{p r}^{B}\right\}_{n}$. So form factor $F_{N}^{\alpha}$ is a linear function of each $\ell\left(\lambda_{j}^{C}\right)$ and a linear function of each $\ell^{-1}\left(\lambda_{j}^{B}\right)$. Coefficient $\mathbb{R}_{N}$ (part) does not depend on $\ell$ and is a rational 
function of all $\lambda$ 's:

$$
\mathbb{R}_{N}(\text { part })=\mathbb{R}_{N}\left(\begin{array}{ll}
\left\{\lambda_{p r}^{C}\right\}_{n} ; & \left\{\lambda_{a b}^{C}\right\}_{N-n} \\
\left\{\lambda_{p r}^{B}\right\}_{n} ; & \left\{\lambda_{a b}^{B}\right\}_{N-n}
\end{array}\right) .
$$

The proof of (5.1) is straightforward but rather tiresome. It is given in Appendix B.

Our next step is to study the properties of coefficients $\mathbb{R}_{N}$. It is proved in Appendix B that they are represented in the following "factorized" form:

$$
\begin{aligned}
& \mathbb{R}_{N}\left(\begin{array}{ll}
\left\{\lambda_{p r}^{C}\right\}_{n} ; & \left\{\lambda_{a b}^{C}\right\}_{N-n} \\
\left\{\lambda_{p r}^{B}\right\}_{n} ; & \left\{\lambda_{a b}^{B}\right\}_{N-n}
\end{array}\right)=\sigma_{n}^{\alpha}\left(\left\{\lambda_{p r}^{C}\right\}_{n},\left\{\lambda_{p r}^{B}\right\}_{n}\right) \\
& \quad \times \sigma_{N-n}^{\alpha}\left(\left\{\lambda_{a b}^{B}\right\}_{N-n},\left\{\lambda_{a b}^{C}\right\}_{N-n}\right)\left\{\prod_{p r} \prod_{a b} f\left(\lambda_{p r}^{C}, \lambda_{a b}^{C}\right)\right\}\left\{\prod_{p r} \prod_{a b} f\left(\lambda_{a b}^{B}, \lambda_{p r}^{B}\right)\right\} .
\end{aligned}
$$

Product $\prod_{p r} \prod_{a b}$ denotes the independent products over all $\lambda \in\left\{\lambda_{p r}\right\}$ and all $\lambda \in\left\{\lambda_{a b}\right\}$; this product contains $n(N-n)$ factors. Rational functions $\sigma_{n}^{\alpha}(n=0,1,2, \ldots)$ are uniquely defined by the following five properties:

(1) $\sigma_{n}^{\alpha}$ is a rational function of $2 n$ momenta:

$$
\sigma_{n}^{\alpha}=\sigma_{n}^{\alpha}\left(\left\{\lambda^{C}\right\}_{n},\left\{\lambda^{B}\right\}_{n}\right)
$$

(2) It is a symmetrical function of $\lambda_{j}^{C}(j=1, \ldots, n)$ and a symmetrical function of $\lambda_{k}^{B}(k=1, \ldots, n)$.

(3) For $n \geqq 1$ it decreases as $1 / \lambda_{j}^{B}$ at $\lambda_{j}^{B} \rightarrow \infty$ and all other $\lambda^{B}$ and $\lambda^{C}$ fixed. It also decreases as $1 / \lambda_{j}^{C}$ at $\lambda_{j}^{C} \rightarrow \infty$ and all other $\lambda^{C}$ and $\lambda^{B}$ fixed.

(4) The only singularities of functions $\sigma_{n}^{\alpha}$ are first-order poles at $\lambda_{j}^{C} \rightarrow \lambda_{k}^{B}(j, k$ $=1, \ldots, n)$. The residue at the pole is expressed in terms of $\sigma_{n-1}^{\alpha} ;$ as $\lambda_{n}^{C} \rightarrow \lambda_{n}^{B} \rightarrow \lambda_{n}$ one has

$$
\begin{aligned}
& \left.\sigma_{n}^{\alpha}\left(\left\{\lambda^{C}\right\}_{n},\left\{\lambda^{B}\right\}_{n}\right)\right|_{\lambda_{n}^{C} \rightarrow \lambda_{n}^{B}} \\
& =\frac{i c}{\lambda_{n}^{C}-\lambda_{n}^{B}}\left\{e^{\alpha} \prod_{j=1}^{n-1} f_{j n}^{C} f_{n j}^{B}-\prod_{j=1}^{n-1} f_{n j}^{C} f_{j n}^{B}\right\} \sigma_{n-1}^{\alpha}\left(\left\{\lambda^{C}\right\}_{n-1},\left\{\lambda^{B}\right\}_{n-1}\right) .
\end{aligned}
$$

Here as usual $f_{j n}^{C, B} \equiv f\left(\lambda_{j}^{C, B}-\lambda_{n}\right)$. There are no $\lambda_{n}^{C, B}$ at $\sigma_{n-1}^{\alpha}$ at the right-hand side here. The residues of $\sigma_{n}^{\alpha}$ at $\lambda_{j}^{C} \rightarrow \lambda_{k}^{B}$ for other $j, k$ can be easily restored from (5.5) due to symmetry property (2).

Notice that due to properties (1)-(4) function $\sigma_{n}^{\alpha}$ can be represented in the form

$$
\sigma_{n}^{\alpha}\left(\left\{\lambda^{C}\right\}_{n},\left\{\lambda^{B}\right\}_{n}\right)=\frac{\pi_{n}^{\alpha}\left(\left\{\lambda^{C}\right\}_{n},\left\{\lambda^{B}\right\}_{n}\right)}{\prod_{j=1}^{n} \prod_{k=1}^{n}\left(\lambda_{j}^{B}-\lambda_{k}^{C}\right)} .
$$

Here $\pi_{n}^{\alpha}$ is a polynomial in each of $\lambda$; the degree of the polynomial in given $\lambda$ being $n-1$ at all the other $\lambda$ fixed.

(5) By definition $\sigma_{0}^{\alpha} \equiv 1$.

Functions $\sigma_{n}^{\alpha}$ thus defined exist and are defined uniquely. It is proved in Appendix B. These functions can be calculated by recursion using these properties; 
the first function, for example, is $\sigma_{1}^{\alpha}\left(\lambda^{C} ; \lambda^{B}\right)=g\left(\lambda^{C}, \lambda^{B}\right)[\exp (\alpha)-1]$. The following property will be of further importance

$$
\sigma_{n}^{\alpha}\left(\left\{\lambda^{B}\right\}_{n},\left\{\lambda^{C}\right\}_{n}\right)=\exp \{n \alpha\} \sigma_{n}^{-\alpha}\left(\left\{\lambda^{C}\right\}_{n},\left\{\lambda^{B}\right\}_{n}\right) .
$$

Formulae (5.1)-(5.3) give the representation for form factor $F_{N}^{\alpha}$ which appears to be very useful in investigating properties of irreducible parts.

\section{Form Factor of Operator $\mathbf{Q}_{1}$}

Our aim is to investigate the irreducible part of the mean value of operator $\mathbf{Q}_{1}^{2}$. To do this one has to study the form factor $F_{N}^{\prime}$ of operator $\mathbf{Q}_{1}$ :

$$
F_{N}^{\prime}\left(\left\{\lambda^{C}\right\}_{N},\left\{\lambda^{B}\right\}_{N},\left\{\ell^{C}\right\}_{N},\left\{\ell^{B}\right\}_{N}\right) \equiv\left\langle\psi_{N}\left(\lambda_{1}^{C} \ldots \lambda_{N}^{C}\right)\left|\mathbf{Q}_{1}\right| \psi_{N}\left(\lambda_{1}^{B} \ldots \lambda_{N}^{B}\right)\right\rangle .
$$

Here $\psi_{N}$ are eigenfunctions (2.10), (2.15). Form factor $F_{N}^{\prime}$ is easily expressed in terms of $F_{N}^{\alpha}(4.16)$ :

$$
F_{N}^{\prime}=\partial F_{N}^{\alpha} /\left.\partial \alpha\right|_{\alpha=0}
$$

The orthogonality of eigenfunctions for different sets of $\lambda$ leads to the property: $\left.F_{N}^{\alpha}\right|_{\alpha=0}=\delta_{N 0}$. Considering representation (5.1)-(5.3) one concludes:

$$
\left.\sigma_{n}^{\alpha}\right|_{\alpha=0}=\delta_{n 0} .
$$

Differentiating representation (5.1)-(5.3) with respect to $\alpha$ at $\alpha=0$, one then obtains:

$$
F_{N}^{\prime}=\left\{\prod_{j=1}^{N} \ell\left(\lambda_{j}^{C}\right) \prod_{j=1}^{N} \ell^{-1}\left(\lambda_{j}^{B}\right)-1\right\} \sigma_{N}^{\prime}\left(\left\{\lambda^{C}\right\}_{N},\left\{\lambda^{B}\right\}_{N}\right),
$$

where

$$
\sigma_{N}^{\prime}\left(\left\{\lambda^{C}\right\}_{N},\left\{\lambda^{B}\right\}_{N}\right) \equiv \partial \sigma_{N}^{\alpha}\left(\left\{\lambda^{C}\right\}_{N},\left\{\lambda^{B}\right\}_{N}\right) /\left.\partial \alpha\right|_{\alpha=0}
$$

(see (5.3)). Here we use the property $\sigma_{N}^{\prime}\left(\left\{\lambda^{C}\right\},\left\{\lambda^{B}\right\}\right)=-\sigma_{N}^{\prime}\left(\left\{\lambda^{B}\right\},\left\{\lambda^{C}\right\}\right)$ which follows from (5.7).

Properties of functions $\sigma_{n}^{\prime}$ is restored from properties of functions $\sigma_{n}^{\alpha}$ discussed in the previous section:

(1) $\sigma_{n}^{\prime}$ is a rational function of $2 n$ variables $\left\{\lambda^{C}\right\}_{n},\left\{\lambda^{B}\right\}_{n}$.

(2) It is a symmetrical function of $\lambda_{j}^{C}$ and of $\lambda_{k}^{B}$ (separately).

(3) It decreases as $1 / \lambda$ in given $\lambda$ when all the other $\lambda$ 's are fixed.

(4) The only singularities of functions $\sigma_{n}^{\prime}$ are first-order poles at $\lambda_{j}^{C} \rightarrow \lambda_{k}^{B}(j, k$ $=1, \ldots, n)$. If $\lambda_{n}^{C} \rightarrow \lambda_{n}^{B} \rightarrow \lambda_{n}$, one has

$$
\begin{aligned}
& \left.\sigma_{n}^{\prime}\left(\left\{\lambda^{C}\right\}_{n},\left\{\lambda^{B}\right\}_{n}\right)\right|_{\lambda_{n}^{C} \rightarrow \lambda_{n}^{B}} \\
& \quad=\frac{i C}{\lambda_{n}^{C}-\lambda_{n}^{B}}\left\{\prod_{j=1}^{n-1} f_{j n}^{C} f_{n j}^{B}-\prod_{j=1}^{n-1} f_{n j}^{C} f_{j n}^{B}\right\} \sigma_{n-1}^{\prime}\left(\left\{\lambda^{C}\right\}_{n-1},\left\{\lambda^{B}\right\}_{n-1}\right) .
\end{aligned}
$$

(5) For small $n$ one has: $\sigma_{0}^{\prime}=0 ; \sigma_{1}^{\prime}\left(\lambda^{C} ; \lambda^{B}\right)=g\left(\lambda^{C}, \lambda^{B}\right)$;

$$
\sigma_{2}^{\prime}\left(\left\{\lambda_{1}^{C} \lambda_{2}^{C}\right\},\left\{\lambda_{1}^{B} \lambda_{2}^{B}\right\}\right)=2 i c^{3}\left(\lambda_{1}^{B}+\lambda_{2}^{B}-\lambda_{1}^{C}-\lambda_{2}^{C}\right) \prod_{j, k=1}^{2}\left(\lambda_{j}^{C}-\lambda_{k}^{B}\right)^{-1} .
$$

These properties define $\sigma_{n}^{\prime}$ uniquely and can be used as a practical tool for 
calculation of $\sigma_{n}^{\prime} ; \sigma_{2}^{\prime}$ was calculated in this way. The asymptotics in the coupling constant at $\lambda$ fixed are especially easy to obtain. At $c \rightarrow \infty$ one has

$$
\left.\sigma_{N}^{\prime}\left(\left\{\lambda^{C}\right\}_{N},\left\{\lambda^{B}\right\}_{N}\right)\right|_{c \rightarrow \infty}=-\frac{2^{N-1}(i c)^{N(N-1)+1}\left(\sum^{N} \lambda_{j}^{B}-\sum^{N} \lambda_{j}^{C}\right)^{N-1}}{\prod_{j, k=1}^{N}\left(\lambda_{j}^{B}-\lambda_{k}^{C}\right)} \sim c^{N(N-1)+1}
$$

at $c \rightarrow 0$

$$
\begin{aligned}
& \left.\sigma_{N}^{\prime}\left(\left\{\lambda^{C}\right\}_{N},\left\{\lambda^{B}\right\}_{N}\right)\right|_{c \rightarrow 0} \\
& \quad=\frac{i}{N c}\left(\sum_{j=1}^{N} \lambda_{j}^{B}-\sum_{j=1}^{N} \lambda_{j}^{C}\right) \sum_{P, Q} \prod_{n=1}^{N} g\left(\lambda_{P_{n}}^{B}, \lambda_{Q_{n}}^{C}\right) g\left(\lambda_{P_{n+1}}^{B}, \lambda_{Q_{n}}^{C}\right) \sim c^{2 N-1} .
\end{aligned}
$$

Here the sum is over two independent permutations $P$ and $Q$ of $n$ numbers.

\section{Irreducible Parts in the Generalized Model}

Consider the "normalized" mean value of operator $\exp \left\{\alpha \mathbf{Q}_{1}\right\}$ with respect to the eigenfunction (see (4.12)):

$$
\left\langle\exp \left\{\alpha \mathbf{Q}_{1}\right\}\right\rangle_{N} \equiv c^{-N}\left(\prod_{j \neq k}^{N} f_{j k}\right)^{-1} \mathscr{M}_{N}^{\alpha}\left(\{\lambda\}_{N},\{x\}_{N},\{y\}_{N},\{\ell\}_{N}\right) .
$$

Let us write down explicitly the independent variables

$$
\left\langle\exp \left\{\alpha \mathbf{Q}_{1}\right\}\right\rangle_{N}=\left\langle\exp \left\{\alpha \mathbf{Q}_{1}\right\}\right\rangle_{N}\left(\{\lambda\}_{N},\{x\}_{N},\{y\}_{N},\{\ell\}_{N}\right) .
$$

The value of (7.2) at $x_{j}=y_{j}=0$ we call the irreducible part $I_{N}^{\alpha}$ of $\left\langle\exp \left\{\alpha \mathbf{Q}_{1}\right\}\right\rangle_{N}$ :

$$
I_{N}^{\alpha}=I_{N}^{\alpha}\left(\{\lambda\}_{N},\{l\}_{N}\right)=\left.\left\langle\exp \left\{\alpha \mathbf{Q}_{1}\right\}\right\rangle_{N}\right|_{x_{j}=y_{j}=0(j=1, \ldots, N)} .
$$

"Normalized" mean values of operators $\mathbf{Q}_{1}^{m}(m=0,1,2, \ldots)$ are defined in terms of generating mean value (7.1) as

$$
\left\langle\mathbf{Q}_{1}^{m}\right\rangle_{N}=\partial^{m}\left\langle\exp \left\{\alpha \mathbf{Q}_{1}\right\}\right\rangle_{N} /\left.\partial \alpha^{m}\right|_{\alpha=0} .
$$

The irreducible part of $\left\langle\mathbf{Q}_{1}^{m}\right\rangle_{N}$ is generated by $I_{N}^{\alpha}(7.3)$ :

$$
I_{N}^{(m)} \equiv \partial^{m} I_{N}^{\alpha} /\left.\partial \alpha^{m}\right|_{\alpha=0}
$$

The mean value $\langle 1\rangle_{N}$ of the unit operator is already calculated $(2.16):\langle 1\rangle_{N}$ $=\operatorname{det}_{N}\left(\varphi^{\prime}\right)$. The corresponding irreducible part $I_{N}^{(0)}$ is equal to

$$
I_{N}^{(0)}=\delta_{N 0} \text {. }
$$

Irreducible parts $I_{N}^{(m)}(m \geqq 1)$ are rather difficult to calculate starting directly from their definition. So we give a method of calculation in terms of form factor (4.16), (6.1): 


$$
\begin{aligned}
& I_{N}^{\alpha}\left(\left\{\lambda_{j}\right\}_{N},\left\{\ell_{j}\right\}_{N}\right)=c^{-N}\left(\prod_{j \neq k} f_{j k}\right)^{-1} \\
& \quad \times \lim _{\varepsilon \rightarrow 0} F_{N}^{\alpha}\left(\left\{\lambda_{j}^{C}=\lambda_{j}\right\}_{N},\left\{\lambda_{j}^{B}=\lambda_{j}+\varepsilon\right\}_{N},\left\{\ell_{j}^{C}=\ell_{j}\right\}_{N},\left\{\ell_{j}^{B}=\ell_{j}\right\}_{N}\right) .
\end{aligned}
$$

This formula explains why we studied in detail the form factor in previous sections and is proved in Appendix C. Equation (7.7) is applied below to investigate and calculate irreducible parts. For example it is quite obvious from (6.4) and (7.7) that

$$
I_{N}^{(1)}=0 \quad(N=0,1,2, \ldots) .
$$

Of most interest for us is the irreducible part $I_{N}^{(2)}$ which is much more complicated. Further we denote it simply $I_{N}^{(2)} \equiv I_{N}$, suppressing the superscript. It is obvious that for the NS-model definitions (7.3), (7.5) for $m=2$ lead to (1.15). The irreducible part $I_{N}$ of $\left\langle\mathbf{Q}_{1}^{2}\right\rangle_{N}$ does not vanish for $N \geqq 2$. To investigate it let us study the form factor of operator $\mathbf{Q}_{1}^{2}$ :

$$
\begin{aligned}
F_{N}^{\prime \prime}\left(\left\{\lambda^{C}\right\},\left\{\lambda^{B}\right\},\left\{\ell^{C}\right\},\left\{\ell^{B}\right\}\right) & \equiv\left\langle\psi_{N}\left(\lambda_{1}^{C} \ldots \lambda_{N}^{C}\right)\left|\mathbf{Q}_{1}^{2}\right| \psi_{N}\left(\lambda_{1}^{B} \ldots \lambda_{N}^{B}\right)\right\rangle \\
& =\partial^{2} F_{N}^{\alpha} /\left.\partial \alpha^{2}\right|_{\alpha=0} .
\end{aligned}
$$

Using representation (5.1)-(5.3) for $F_{N}^{\alpha}$ and Eq. (6.3), one obtains:

$$
\begin{aligned}
F_{N}^{\prime \prime}= & \sigma_{N}^{\prime \prime}\left(\left\{\lambda^{B}\right\}_{N},\left\{\lambda^{C}\right\}_{N}\right)+\left(\prod_{j=1}^{N} \ell\left(\lambda_{j}^{C}\right) \ell^{-1}\left(\lambda_{j}^{B}\right)\right) \sigma_{N}^{\prime \prime}\left(\left\{\lambda^{C}\right\}_{N},\left\{\lambda^{B}\right\}_{N}\right) . \\
& +2 \sum_{p a r t}^{N-1 \geqq n \geqq 1} \sigma_{n}^{\prime}\left(\left\{\lambda_{p r}^{C}\right\}_{n},\left\{\lambda_{p r}^{B}\right\}_{n}\right) \sigma_{N-n}^{\prime}\left(\left\{\lambda_{a b}^{B}\right\}_{N-n},\left\{\lambda_{a b}^{C}\right\}_{N-n}\right) \\
& \times\left\{\prod_{p r} \ell\left(\lambda_{p r}^{C}\right) \ell^{-1}\left(\lambda_{p r}^{B}\right)\right\}\left\{\prod_{p r} \prod_{a b} f\left(\lambda_{p r}^{C}, \lambda_{a b}^{C}\right)\right\}\left\{\prod_{p r a b} f\left(\lambda_{a b}^{B}, \lambda_{p r}^{B}\right)\right\} .
\end{aligned}
$$

The sum here is taken as is explained after (5.1) but we have written down explicitly the two terms corresponding to partition $\left\{\lambda_{p r}^{C}\right\}=\varnothing ;\left\{\lambda_{p r}^{B}\right\}=\varnothing$ and to partition $\left\{\lambda_{p r}^{C}\right\}=\left\{\lambda^{C}\right\}_{N} ;\left\{\lambda_{p r}^{B}\right\}=\left\{\lambda^{B}\right\}_{N}$. We denote card $\left\{\lambda_{p r}^{C}\right\}_{n}=\operatorname{card}\left\{\lambda_{p r}^{B}\right\}_{n}=n$ and $\sigma_{n}^{\prime \prime} \equiv$ $\partial^{2} \sigma_{N}^{\alpha} /\left.\partial \alpha^{2}\right|_{\alpha=0}$.

Now we can investigate the irreducible part $I_{N}$ using Eq. (7.7). One can see that $I_{N}$ can be represented in the form:

$$
\begin{aligned}
I_{N}\left(\{\lambda\}_{N},\{\ell\}_{N}\right)= & \sum_{\{\lambda\}=\left\{\lambda_{+}\right\} \cup\left\{\lambda_{-}\right\} \cup\left\{\lambda_{0}\right\}}^{0 \leqq n} \prod_{(+)}^{n} \ell\left(\lambda_{+}\right) \prod_{(-)}^{n} \ell^{-1}\left(\lambda_{-}\right) \\
& \times \mathscr{A}_{N}^{n}\left(\left\{\lambda_{+}\right\}_{n},\left\{\lambda_{-}\right\}_{n},\left\{\lambda_{0}\right\}_{N-2 n}\right) .
\end{aligned}
$$

The sum here is taken over all the partitions of the set $\{\lambda\}_{N}$ into three disjoint subsets: $\operatorname{card}\left\{\lambda_{+}\right\}_{n}=\operatorname{card}\left\{\lambda_{-}\right\}_{n}=n ; \operatorname{card}\left\{\lambda_{0}\right\}_{N-2 n}=N-2 n ; 0 \leqq n \leqq[N / 2]$. Coefficients $\mathscr{A}_{N}^{n}$ are the Fourier coefficients of the irreducible part $I_{N}$ (compare (1.16)). They do not depend on $\ell_{j}$ but only on $\lambda_{j}$ being a rational functions of $\lambda$. Fourier coefficients depend on the $R$-matrix only and do not depend on the concrete model. 
All the dependence on concerete models enters through vacuum values $\ell(\lambda)$ and is written in (7.11) explicitly.

Turning now to Eq. (7.7) one sees that the first two terms in (7.10) contribute only to the term with $n=0$ in the sum in (7.11). This term with $n=0$ can be expressed as a linear function of other terms with $n \geqq 1$. Indeed, it is shown in Appendix $\mathrm{D}$ that $I_{N}\left(\{\lambda\}_{N},\left\{\ell_{j}=1\right\}_{N}\right)=0$. Hence one can rewrite (7.11) as follows:

$$
\begin{aligned}
I_{N}\left(\left\{\lambda_{j}\right\}_{N},\left\{\ell_{j}\right\}_{N}\right)= & \sum_{\{\lambda\}=\left\{\lambda_{+}\right\} \cup\left\{\lambda_{-}\right\} \cup\left\{\lambda_{0}\right\}}^{1 \leqq n \leqq[N / 2]}\left\{\prod_{(+)} \ell\left(\lambda_{+}\right) \prod_{(-)} \ell^{-1}\left(\lambda_{-}\right)-1\right\} \\
& \times \mathscr{A}_{N}^{n}\left(\left\{\lambda_{+}\right\}_{n},\left\{\lambda_{-}\right\}_{n},\left\{\lambda_{0}\right\}_{N-2 n}\right),
\end{aligned}
$$

where the sum does not contain coefficient $\mathscr{A}_{N}^{0}$. Coefficients $\mathscr{A}_{N}^{n}(n \geqq 1)$ are expressed through functions $\sigma_{k}^{\prime}$ only. So we do not need functions $\sigma_{k}^{\prime \prime}$ to calculate $I_{N}$. The functions $\sigma_{n}^{\prime}$ defined in the previous section can be calculated rather simply by recurrence. Formulae (7.7) and (7.10) then permit us to calculate the irreducible part $I_{N}$. Irreducible parts $I_{0}=I_{1}=0$. Irreducible parts $I_{2}$ and $I_{3}$ are given in the next section. The computation of $I_{N}(N \geqq 4)$ also is quite straightforward. We could not obtain the simple formula for general $I_{N}$. However one can easily establish the behavior of $I_{N}$ in coupling constant $c$ for $c \rightarrow \infty$ and $c \rightarrow 0$, using formulae (6.8), (6.9), (7.7), (7.10). One obtains

$$
\begin{array}{llll}
I_{N} \sim c^{2-N} & \text { at } & c \rightarrow \infty & (N \geqq 2), \\
I_{N} \sim c^{N-2} & \text { at } & c \rightarrow 0 & (N \geqq 2) .
\end{array}
$$

This remarkable behavior means that $I_{N}$ is small in coupling constant in the weak as well as the strong coupling limit. This is one of the main results of the paper which permits us to construct an effective perturbation theory for correlation functions [11].

Our results are summarized in the next section.

\section{Main Properties of the Mean Value of Operator $Q_{1}^{2}$}

We begin with the mean value of the identity operator with respect to eigenfunctions (2.10) (see (2.16)):

$$
\begin{aligned}
\langle\mathbf{1}\rangle_{N} & \equiv c^{-N}\left(\prod_{j \neq k} f_{j k}\right)^{-1}\left\langle\psi_{N}\left(\lambda_{1} \ldots \lambda_{N}\right) \mid \psi_{N}\left(\lambda_{1} \ldots \lambda_{N}\right)\right\rangle \\
& =c^{-N}\left(\prod_{j=1}^{N} a\left(\lambda_{j}\right) d\left(\lambda_{j}\right) \prod_{k \neq \ell} f_{k t}\right)^{-1}\left\langle 0\left|\prod_{j=1}^{N} C\left(\lambda_{j}\right) \prod_{k=1}^{N} B\left(\lambda_{k}\right)\right| 0\right\rangle \\
& =\operatorname{det}_{N}\left(\varphi^{\prime}\right) .
\end{aligned}
$$

Here $\lambda_{j}(j=1, \ldots, N)$ satisfy s.t.e. (2.12). The irreducible part of the identity operator $I_{N}^{0}=\delta_{N 0}(\operatorname{see}(7.5),(7.6))$.

We now remind the reader of the main properties of the mean value $\left\langle\mathbf{Q}_{1}\right\rangle_{N}$ with 
respect to eigenfunctions:

$$
\begin{aligned}
\left\langle\mathbf{Q}_{1}\right\rangle_{N} & \equiv c^{-N}\left(\prod_{j \neq k} f_{j k}\right)^{-1}\left\langle\psi_{N}\left(\lambda_{1} \ldots \lambda_{N}\right)\left|\mathbf{Q}_{1}\right| \psi_{N}\left(\lambda_{1} \ldots \lambda_{N}\right)\right\rangle \\
& =c^{-N}\left(\prod_{j=1}^{N} a\left(\lambda_{j}\right) d\left(\lambda_{j}\right) \prod_{k \neq \ell} f_{k \ell}\right)^{-1}\left\langle 0\left|\prod_{j=1}^{N} C\left(\lambda_{j}\right) \mathbf{Q}_{1} \prod_{k=1}^{N} B\left(\lambda_{k}\right)\right| 0\right\rangle .
\end{aligned}
$$

It depends on $4 N$ variables: $\left\langle\mathbf{Q}_{1}\right\rangle_{N}=\left\langle\mathbf{Q}_{1}\right\rangle_{N}\left(\{\lambda\}_{N},\{x\}_{N},\{\ell\}_{N}\right.$ and possesses the following properties as a function of these variables:

(1) It is invariant under replacement

$$
\left(\lambda_{k}, x_{k}, y_{k}, \ell_{k}\right) \leftrightarrow\left(\lambda_{j}, x_{j}, y_{j}, \ell_{j}\right) ; \quad k, j=1, \ldots, N
$$

(2) It is a linear function of $x_{N}$ and of $y_{N}$.

(3) The coefficient at $y_{N}$ is equal to

$$
\frac{\partial}{\partial y_{N}}\left\langle\mathbf{Q}_{1}\right\rangle_{N}=\left\langle\mathbf{Q}_{1}\right\rangle_{N-1}\left(\left\{\lambda_{j}\right\}_{N-1},\left\{x_{j}\right\}_{N-1},\left\{y_{j}+K_{j N}\right\}_{N-1},\left\{\ell_{j}\right\}_{N-1}\right),
$$

and the coefficient at $x_{N}$ is equal to

$$
\frac{\partial}{\partial x_{N}}\left\langle\mathbf{Q}_{1}\right\rangle_{N}=\left\langle\mathbf{Q}_{1}+\mathbf{1}\right\rangle_{N-1}\left(\left\{\lambda_{j}\right\}_{N-1},\left\{x_{j}+K_{j N}\right\}_{N-1},\left\{y_{j}\right\}_{N-1},\left\{\tilde{\ell}_{j}\right\}_{N-1}\right),
$$

where $K_{j N}$ and $\tilde{\ell}_{j}$ are defined in (3.9) and (4.3) as

$$
K_{j N}=2 c /\left[\left(\lambda_{j}-\lambda_{N}\right)^{2}+c^{2}\right] ; \quad \tilde{\ell}_{j}=\ell_{j}\left(f_{j N} / f_{N j}\right) .
$$

Here we use the notation $\left\langle\mathbf{Q}_{1}+\mathbf{1}\right\rangle_{N} \equiv\left\langle\mathbf{Q}_{1}\right\rangle_{N}+\langle\mathbf{1}\rangle_{N}$. It should be noted that variables $x_{N}, y_{N}, \ell_{N}$ are absent at the right-hand side of (8.3), (8.4) and $\lambda_{N}$ enters only in $K_{j N}$ and in factors $f_{j N}$ and $f_{N j}$ modifying $\ell$ to $\tilde{\ell}$. These three properties can be easily obtained from (4.12)-(4.15).

(4) The mean value $\left\langle\mathbf{Q}_{1}\right\rangle_{N}$ is equal to zero at $x_{j}=y_{j}=0(j=1, \ldots, N)$ and $\lambda_{k}, \ell_{k}$ fixed: $\left\langle\mathbf{Q}_{1}\right\rangle_{N}\left(\{\lambda\}_{N},\{0\}_{N},\{0\}_{N},\{\ell\}_{N}\right)=0 ; N=0,1,2, \ldots$. This means that the irreducible part of $\left\langle\mathbf{Q}_{1}\right\rangle_{N}$ is equal to zero (see (7.8)):

$$
I_{N}^{(1)}=0 \quad(N=0,1,2, \ldots) .
$$

(5) It can be easily seen that in the one-particle sector $\left\langle\mathbf{Q}_{1}\right\rangle_{1}=x_{1}$.

Now let us turn to the mean value of operator $\mathbf{Q}_{1}^{2}$ :

$$
\begin{aligned}
\left\langle\mathbf{Q}_{1}^{2}\right\rangle_{N} & \equiv c^{-N}\left(\prod_{j \neq k} f_{j k}\right)^{-1}\left\langle\psi_{N}\left(\lambda_{1} \ldots \lambda_{N}\right)\left|\mathbf{Q}_{1}^{2}\right| \psi_{N}\left(\lambda_{1} \ldots \lambda_{N}\right)\right\rangle \\
& =c^{-N}\left(\prod_{J=1}^{N} a\left(\lambda_{j}\right) d\left(\lambda_{j}\right) \prod_{k \neq \ell} f_{k t}\right)^{-1}\left\langle 0\left|\prod_{j=1}^{N} C\left(\lambda_{j}\right) \mathbf{Q}_{1}^{2} \prod_{k=1}^{N} B\left(\lambda_{k}\right)\right| 0\right\rangle,
\end{aligned}
$$


which depends on the same $4 N$ variables

$$
\left\langle\mathbf{Q}_{1}^{2}\right\rangle_{N}=\left\langle\mathbf{Q}_{1}^{2}\right\rangle_{N}\left(\{\lambda\}_{N},\{x\}_{N},\{y\}_{N},\{\ell\}_{N}\right) .
$$

Its properties are as follows.

(1) It is invariant under replacement of

$$
\left(\lambda_{k}, x_{k}, y_{k}, \ell_{k}\right) \leftrightarrow\left(\lambda_{j}, x_{j}, y_{j}, \ell_{j}\right) ; \quad k, j=1, \ldots, N .
$$

(2) It is a linear function of $x_{N}$ and $y_{N}$.

(3) The coefficients at $y_{N}$ and $x_{N}$ are

$$
\begin{aligned}
& \frac{\partial}{\partial y_{N}}\left\langle\mathbf{Q}_{1}^{2}\right\rangle_{N}=\left\langle\mathbf{Q}_{1}^{2}\right\rangle_{N-1}\left(\left\{\lambda_{j}\right\}_{N-1},\left\{x_{j}\right\}_{N-1},\left\{y_{j}+K_{j N}\right\}_{N-1},\left\{\ell_{j}\right\}_{N-1}\right) ; \\
& \frac{\partial}{\partial x_{N}}\left\langle\mathbf{Q}_{1}^{2}\right\rangle_{N}=\left\langle\left(\mathbf{Q}_{1}+\mathbf{1}\right)^{2}\right\rangle_{N}\left(\left\{\lambda_{j}\right\}_{N-1},\left\{x_{j}+K_{j N}\right\}_{N-1},\left\{y_{j}\right\}_{N-1},\left\{\tilde{\ell}_{j}\right\}_{N-1}\right),
\end{aligned}
$$

with the same $K_{j N}$ and $\tilde{\ell}_{j}$ as in (8.3), (8.4). Here we put $\left\langle\left(\mathbf{Q}_{1}+1\right)^{2}\right\rangle_{N}=\left\langle\mathbf{Q}_{1}^{2}\right\rangle_{N}$ $+2\left\langle\mathbf{Q}_{1}\right\rangle_{N}+\langle\mathbf{1}\rangle_{N}$. On the right-hand side of (8.8), (8.9) $x_{N}, y_{N}, \ell_{N}$ are absent and $\lambda_{N}$ enters only in $K_{j N}$ and $f_{j N}, f_{N j}$ modifying $\ell_{j}(j=1, \ldots, N-1)$ to $\bar{\ell}_{j}$. These properties (1)-(3) can be easily obtained from (4.12)-(4.15).

(4) The mean value at $x_{j}=y_{j}=0$ is equal to the irreducible part $I_{N} \equiv I_{N}^{(2)}$ (see $(7.5))$ :

$$
\begin{aligned}
\left\langle\mathbf{Q}_{1}^{2}\right\rangle_{N}\left(\left\{\lambda_{j}\right\}_{N},\left\{x_{j}\right.\right. & \left.=0\}_{N},\left\{y_{j}=0\right\}_{N},\left\{\ell_{j}\right\}_{N}\right) \\
& =I_{N}\left(\left\{\lambda_{j}\right\}_{N},\left\{\ell_{j}\right\}_{N}\right),
\end{aligned}
$$

which was studied in Sect. 7.

(5) In the one-particle sector $\left\langle\mathbf{Q}_{1}^{2}\right\rangle_{1}=x_{1}$.

Remember now properties of the irreducible part $I_{N}$. It was shown in Sect. 7 that it can be represented in the form (7.11), (7.12). The function $I_{N}$ is symmetric under replacement of pairs $\left(\lambda_{k}, \ell_{k}\right) \rightarrow\left(\lambda_{j}, \ell_{j}\right)$. If $\ell^{*}\left(\lambda^{*}\right)=\ell^{-1}(\lambda)$ (as for the NS-model), then $I_{N}$ is real at $\lambda_{j}$ real $(j=1, \ldots, N)$. In Sect. 7 important properties (7.13), (7.14) concerning the asymptotics of the irreducible parts in the coupling constant were proved. The methods of calculation of $I_{N}$ were discussed in Sect. 1 and in Sect. 7, which is especially simple. By means of these methods $I_{N}$ can be easily calculated for small $N$; for example, $I_{0}=I_{1}=0$;

$$
\begin{aligned}
I_{2}\left(\left\{\lambda_{1} \lambda_{2}\right\},\left\{\ell_{1} \ell_{2}\right\}\right) & =\mathscr{A}_{2}^{1}\left(\lambda_{1} \lambda_{2}\right)\left(\ell_{1} \ell_{2}^{-1}-1\right)+\mathscr{A}_{2}^{1}\left(\lambda_{2} \lambda_{1}\right)\left(\ell_{2} \ell_{1}^{-1}-1\right) \\
\mathscr{A}_{2}^{1}\left(\lambda_{1} \lambda_{2}\right) & =-\frac{2}{\lambda_{12}^{2}}\left(\frac{\lambda_{12}+i c}{\lambda_{12}-i c}\right) .
\end{aligned}
$$

The irreducible part $I_{3}$ is equal to

$$
I_{3}\left(\left\{\lambda_{1} \lambda_{2} \lambda_{3}\right\},\left\{\ell_{1} \ell_{2} \ell_{3}\right\}\right)=\sum_{P} \mathscr{A}_{3}^{1}\left(\lambda_{P_{1}} \lambda_{P_{2}} \lambda_{P_{3}}\right)\left[\ell\left(\lambda_{P_{1}}\right) \ell^{-1}\left(\lambda_{P_{2}}\right)-1\right] .
$$

The sum here is taken over all the permutations of $\lambda_{1}, \lambda_{2}, \lambda_{3}$. The Fourier coefficient $\mathscr{A}_{3}^{1}$ is equal to

$$
\mathscr{A}_{3}^{1}\left(\lambda_{1} \lambda_{2} \lambda_{3}\right)=\frac{8 c}{\lambda_{12}^{2}}\left(\frac{\lambda_{12}+i c}{\lambda_{12}-i c}\right)\left[\frac{\lambda_{32}}{\lambda_{31}}+\frac{\lambda_{31}}{\lambda_{32}}\right] \frac{1}{\left(\lambda_{31}+i c\right)\left(\lambda_{23}+i c\right)} .
$$


It should be noted that the properties presented in this section permit us to restore the mean value $\left\langle\mathbf{Q}_{1}^{2}\right\rangle_{N}$ in terms of the irreducible parts $I_{k} ; k \leqq N$ [11]. Let us remind the reader that for the NS-model $r(\lambda)=\exp \{-i \lambda L\} ; \ell(\lambda)=\exp \{-i \lambda x\}$; $m(\lambda)=\exp \{-i \lambda y\} ; z(\lambda)=L ; x(\lambda)=x ; y(\lambda)=y=L-x$.

\section{Conclusion}

So we have demonstrated that the two-site generalized model permits a formulation of the problem of calculation of correlation functions of currents in the frame of QISM. It should be emphasized that our approach can be applied to the calculation of any correlation function. To do this one has to use the generalized model with more than two sites. For example, one can calculate the field correlator $\left\langle\psi(x) \psi^{+}(y)\right\rangle$ in the NS-model by means of a 4-site generalized model, representing the monodromy matrix in the form $T(\lambda)=T_{2}(\lambda) L_{x}(\lambda) T_{1}(\lambda) L_{y}(\lambda)$ (compare with (2.5)). Here $L(\lambda)$ is the local $L$-operator for the NS-model [1]. The correlation function of currents is special in two aspects. It is connected with the simplest two-site model, and its irreducible parts are small in the strong coupling limit (7.13) (for the field correlator this is not the case).

In our paper we considered the XXX case only. It should be mentioned that the generalization to the XXZ-case is quite obvious. So this approach gives the opportunity to calculate the correlation function for the XXZ Heisenberg model and for the sine-Gordon model.

\section{Appendix A}

Let us consider the state

$$
\prod_{j=1}^{N} \mathbb{B}\left(\lambda_{j}\right)|0\rangle \cdot
$$

Here all $\lambda_{j}$ are independent and s.t.e. (2.12) is not supposed to be satisfied. By means of the formula

$$
\mathbb{B}(\lambda)=\left[A_{2}(\lambda) / d_{2}(\lambda)\right] \mathbb{B}_{1}(\lambda)+\left[D_{1}(\lambda) / d_{1}(\lambda)\right] \mathbb{B}_{2}(\lambda),
$$

we present this state in terms of states $\prod \mathbb{B}_{1}(\lambda)|0\rangle$ and $\prod \mathbb{B}_{2}(\lambda)|0\rangle$. The generalization of standard arguments of QISM $[1,18]$ shows that

$$
\begin{aligned}
\prod_{j=1}^{N} \mathbb{B}\left(\lambda_{j}\right)|0\rangle= & \sum_{\{\lambda\}=\left\{\lambda_{I}\right\} \cup\left\{\lambda_{I I}\right\}}^{n_{1}+n_{2}=N}\left(\prod_{I} \prod_{I I} m\left(\lambda_{I}\right) f\left(\lambda_{I}, \lambda_{I I}\right)\right) \\
& \times\left(\prod_{I I} \mathbb{B}_{2}\left(\lambda_{I I}\right)\right)\left(\prod_{I} \mathbb{B}_{1}\left(\lambda_{I}\right)\right)|0\rangle
\end{aligned}
$$

Here the sum is taken over all the partitions of the set $\{\lambda\}_{N}$ into two disjoint subsets $\left\{\lambda_{I}\right\}_{n_{1}}$ and $\left\{\lambda_{I I}\right\}_{n_{2}} ; \operatorname{card}\left\{\lambda_{I}\right\}_{n_{1}}=n_{1} ; \operatorname{card}\left\{\lambda_{I I}\right\}_{n_{2}}=n_{2}$ and $n_{1}+n_{2}=\operatorname{card}\{\lambda\}_{N}=N$. Product $\prod_{I} \prod_{I I}$ is an independent product over all $\lambda \in\left\{\lambda_{I}\right\}$ and $\lambda \in\left\{\lambda_{I I}\right\}$, and thus contains $n_{1} n_{2}$ factors.

Formula (A.3) permits us to calculate the action of operator $\exp \left\{\alpha \mathbf{Q}_{1}\right\}$ on state (A.1): 


$$
\begin{aligned}
& \exp \left\{\alpha \mathbf{Q}_{1}\right\} \prod_{j=1}^{N} \mathbb{B}\left(\lambda_{j}\right)|0\rangle=\sum_{\{\lambda\}=\left\{\lambda_{I}\right\} \cup\left\{\lambda_{I I}\right\}}^{n_{1}+n_{2}=N} \exp \left\{\alpha n_{1}\right\} \\
& \quad \times\left(\prod_{I} \prod_{I I} m\left(\lambda_{I}\right) f\left(\lambda_{I}, \lambda_{I I}\right)\right)\left(\prod_{I I} \mathbb{B}_{2}\left(\lambda_{I I}\right)\right)\left(\prod_{I} \mathbb{B}_{1}\left(\lambda_{I}\right)\right)|0\rangle
\end{aligned}
$$

Similar representation can be obtained for the state

$$
\begin{aligned}
\langle 0| \prod_{j=1}^{N} \mathbb{C}\left(\lambda_{j}\right): & \\
\langle 0| \prod_{j=1}^{N} \mathbb{C}\left(\lambda_{j}\right)= & \sum_{\{\lambda\}=\left\{\lambda_{1}\right\} \cup\left\{\lambda_{I I}\right\}}^{n_{1}+n_{2}=N}\langle 0|\left(\prod_{I} \mathbb{C}_{1}\left(\lambda_{I}\right)\right)\left(\prod_{I I} \mathbb{C}_{2}\left(\lambda_{I I}\right)\right) \\
& \times\left(\prod_{I} \prod_{I I} f\left(\lambda_{I I}, \lambda_{I}\right) \ell\left(\lambda_{I I}\right)\right) .
\end{aligned}
$$

Combining these two formulae one gets representation (2.19).

It is remarkable that representation (A.3) permits us to find eigenfunctions and eigenvalues of the translation operator $\mathbf{O}$ which is defined for the two-site model as follows (see (2.5), (2.1)):

$$
\mathbf{O} T_{2}(\lambda) T_{1}(\lambda) \mathbf{O}^{-1}=T_{1}(\lambda) T_{2}(\lambda) \equiv \tilde{T}(\lambda)=\left(\begin{array}{ll}
\tilde{A}(\lambda) ; & \tilde{B}(\lambda) \\
\tilde{C}(\lambda) ; & \tilde{D}(\lambda)
\end{array}\right) .
$$

Notice that $\mathbf{O}$ is a scalar quantum operator and $\mathbf{O}|0\rangle=|0\rangle$. Vacuum eigenvalues of matrix $\tilde{T}(\lambda)$ are the same $a(\lambda)$ and $d(\lambda)$ as the ones of matrix $T(\lambda)(2.8)$. Let us consider the state

$$
\begin{aligned}
\mathbf{O} \prod_{j=1}^{N} \mathbb{B}\left(\lambda_{j}\right)|0\rangle & =\prod_{j=1}^{N} \widehat{\mathbb{B}}\left(\lambda_{j}\right)|0\rangle \equiv\left|\hat{\psi}_{N}\left(\lambda_{1} \ldots \lambda_{N}\right)\right\rangle \\
& =\mathbf{O}\left|\psi_{N}\left(\lambda_{1} \ldots \lambda_{N}\right)\right\rangle .
\end{aligned}
$$

If momenta $\lambda_{j}$ satisfy s.t.e. (2.12) it is the eigenstate $\left|\bar{\psi}_{N}\left(\lambda_{1} \ldots \lambda_{N}\right)\right\rangle$ of the trace $\tilde{\tau}(\lambda)=\tilde{A}(\lambda)+\tilde{D}(\lambda)$ of matrix $\tilde{T}(\lambda)$ with the same eigenvalue (2.14) as the state $\left.\psi_{N}\left(\lambda_{1} \ldots \lambda_{N}\right)\right\rangle(2.10)$ is an eigenstate of $\tau(\lambda)=A(\lambda)+D(\lambda)$. The state $\left|\tilde{\psi}_{N}\left(\lambda_{1} \ldots \lambda_{N}\right)\right\rangle$ also can be represented in the form (A.3). By means of s.t.e.(2.12) one can see that the states (2.10) and (A.7) are proportional

$$
\begin{aligned}
\left|\hat{\psi}_{N}\left(\lambda_{1} \ldots \lambda_{N}\right)\right\rangle & =k_{N}\left|\psi_{N}\left(\lambda_{1} \ldots \lambda_{N}\right)\right\rangle ; \\
k_{N} & =\prod_{j=1}^{N} \ell\left(\lambda_{j}\right) .
\end{aligned}
$$

One can easily show also that if $\prod_{j=1}^{N} \tilde{\mathbb{B}}\left(\lambda_{j}\right)|0\rangle=$ Const $\prod_{j=1}^{N} \mathbb{B}\left(\lambda_{j}\right)|0\rangle$, then the s.t.e. (2.12) is valid and Const is given by (A.9). It is also true that

$$
\left\langle\tilde{\psi}_{N}\left(\lambda_{1} \ldots \lambda_{N}\right)\right|=k_{N}^{-1}\left\langle\psi_{N}\left(\lambda_{1} \ldots \lambda_{N}\right)\right| \text {, }
$$

with just the same $k_{N}$. The generalization for an $N$-site model is also quite obvious. 


\section{Appendix B}

Consider first the proof of structure in $\ell$ (5.1). The main points of the proof are as follows:

(i) It is obvious from (3.2), (2.19) that the dependence of $F$ on each individual $\ell\left(\lambda_{j}\right)$ is of the form $F_{N}^{\alpha}=\mathscr{A}_{j} \ell\left(\lambda_{j}\right)+\mathscr{B}_{j}+\mathscr{C}_{j} \ell^{-1}\left(\lambda_{j}\right)$, where $\mathscr{A}_{j}, \mathscr{B}_{j}, \mathscr{C}_{j}$ do not depend on $\ell\left(\lambda_{j}\right)$. Hence one can write

$$
F_{N}^{\alpha}=\sum_{\text {part }} \prod_{(+)} \ell\left(\lambda_{+}\right) \prod_{(-)} \ell^{-1}\left(\lambda_{-}\right) \mathbb{R}_{N}(\text { part }),
$$

where the sum is over all the partitions of set $\left\{\lambda^{B}\right\}_{N} \cup\left\{\lambda_{N}^{C}\right\}$ into disjoint sets $\left\{\lambda_{+}\right\}_{n_{+}} ;\left\{\lambda_{-}\right\}_{n_{-}}$and $\left\{\lambda_{0}\right\}_{n_{0}} ; n_{+}+n_{-}+n_{0}=2 N$.

(ii) Using the fact that $R$-matrix (2.3) commutes with matrix $\varepsilon \otimes \varepsilon$, where

$$
\boldsymbol{\varepsilon}=\left(\begin{array}{ll}
\varepsilon, & 0 \\
0, & \varepsilon^{-1}
\end{array}\right),
$$

one can show that $F_{N}^{\alpha}\left(\left\{\lambda_{j}^{C}\right\},\left\{\lambda_{j}^{B}\right\},\left\{\varepsilon^{-2} \ell_{j}^{C}\right\},\left\{\varepsilon^{-2} \ell_{j}^{B}\right\}\right)=F_{N}^{\alpha}\left(\left\{\lambda_{j}^{C}\right\},\left\{\lambda_{j}^{B}\right\},\left\{\ell_{j}^{C}\right\},\left\{\ell_{j}^{B}\right\}\right)$. Noting that $\ell_{j}^{B, C}$ are independent variables, one then obtains that in (B.1) card $\left\{\lambda_{+}\right\}$ $=\operatorname{card}\left\{\lambda_{-}\right\}$, i.e. $n_{+}=n_{-}$.

(iii) Considering monodromy matrix $\widetilde{T}(\lambda)$ (A.6) and using formulae (A.8), (A.10) for eigenfunctions one comes to the relation

$$
\begin{aligned}
& \left\langle\psi_{N}\left(\lambda_{1}^{C} \ldots \lambda_{N}^{C}\right)\left|\exp \left\{\alpha \mathbf{Q}_{1}\right\}\right| \psi_{N}\left(\lambda_{1}^{B} \ldots \lambda_{N}^{B}\right)\right\rangle \\
& \quad=\left\langle\tilde{\psi}_{N}\left(\lambda_{1}^{C} \ldots \lambda_{N}^{C}\right)\left|\exp \left\{\alpha \mathbf{Q}_{1}\right\}\right| \tilde{\psi}_{N}\left(\lambda_{1}^{B} \ldots \lambda_{N}^{B}\right)\right\rangle \prod_{j=1}^{N} \ell\left(\lambda_{j}^{C}\right) \ell^{-1}\left(\lambda_{j}^{B}\right) .
\end{aligned}
$$

Based on this relation it is easy to prove that in (B.1) set $\left\{\lambda_{+}\right\}$contains only $\lambda^{C}$ 's and set $\left\{\lambda_{-}\right\}$contains only $\lambda^{B}$ 's. Thus representation (5.1) for $F_{N}^{\alpha}$ is proved.

Turn now to the proof of (5.3) which is straightforward but rather lengthy. So we mention only the main points. The idea is to prove (5.3) using induction in $N$.

(i) For $N=1$ the validity of (5.3) is established by direct calculation.

(ii) It is easy to prove that rational function $\mathbb{R}_{N}(5.2)$ is a symmetrical function of all $\lambda$ entering set $\left\{\lambda_{p r}^{C}\right\}$ as well as $\left\{\lambda_{p r}^{B}\right\},\left\{\lambda_{a b}^{C}\right\},\left\{\lambda_{a b}^{B}\right\}$ (separately). So the symmetry properties of the left-hand side of (5.3) under replacement of $\lambda$ 's are the same as of the right hand side.

(iii) Equation (2.19) and properties of scalar products discussed in detail in [4] permit us to establish the structure of singularities of rational function $\mathbb{R}_{N}(5.2)$. One can easily see that the only possible singularities of this function are first order poles of the following two kinds. (a) Poles at $\lambda_{j}-\lambda_{k}=0$ where $\lambda_{j}, \lambda_{k}$ is any pairs of $\lambda$ belonging to the set $\left\{\lambda^{B}\right\}_{N} \cup\left\{\lambda^{C}\right\}_{N}$. (b) Poles at $f\left(\lambda_{j}^{B}, \lambda_{k}^{B}\right)=0$ or $f\left(\lambda_{j}^{C}, \lambda_{k}^{C}\right)=0$ which could occur due to (4.13). However, not all these poles really do occur. Residues at first order poles corresponding to:

$$
\lambda_{a b, j}^{C}=\lambda_{a b, k}^{C} ; \quad \lambda_{p r, j}^{C}=\lambda_{p r, k}^{C} ; \quad \lambda_{a b, j}^{B}=\lambda_{a b, k}^{B} ; \quad \lambda_{p r, j}^{B}=\lambda_{p r, k}^{B}
$$

are equal to zero due to the symmetry property (ii). Residues at all the poles of the kind (b) also appear to be zero which can be shown using Eqs. (A.7), (A.8) of paper [4]. 
So one comes to the statement that the only singularities of the left-hand side of (5.3) are the first-order poles at $\lambda_{p r, j}^{C}=\lambda_{a b, k}^{C} ; \lambda_{p r, j}^{B}=\lambda_{a b, k}^{B} ; \lambda_{p r, j}^{C}=\lambda_{p r, k}^{B} ; \lambda_{a b, j}^{C}=\lambda_{a b, k}^{B}$. Thus the singularities of the left-hand side of (5.3) are the same as of the right-hand side.

(iv) The most subtle point is to prove that $\mathbb{R}_{N}(5.2)$ has zeros at $f\left(\lambda_{p r}^{C}, \lambda_{a b}^{C}\right)=0$ and $f\left(\lambda_{a b}^{B}, \lambda_{p r}^{B}\right)=0$. It can be done using formula (B.2). The form factor at the right-hand side is easily seen to be equal to $\left\langle\tilde{\psi}_{N}^{C}\left|\exp \left\{\alpha \mathbf{Q}_{1}\right\}\right| \tilde{\psi}_{N}^{B}\right\rangle=\exp \{\alpha N\} \times$ $\left\langle\tilde{\psi}_{N}^{C}\left|\exp \left\{-\alpha \mathbf{Q}_{2}\right\}\right| \tilde{\psi}_{N}^{B}\right\rangle=\exp \{\alpha N\} F_{N}^{-\alpha}\left(\left\{\lambda^{C}\right\},\left\{\lambda^{B}\right\},\left\{m^{C}\right\},\left\{m^{B}\right\}\right)$. Here $\mathbf{Q}_{2}$ is a number of particle operator at the second site of the lattice (2.17) and $m(\lambda)$ is defined in (2.9). The representation of the kind (5.1) for $F_{N}^{-\alpha}$ is valid. It should be noted that $m$ 's and not $\ell$ 's enter this representation. Using s.t.e. (4.13) one can, however, return to $\ell$ 's. Comparing now both sides of (B.2) one can see that $\mathbb{R}$ indeed has the required zeros and

$$
\begin{aligned}
\mathbb{R}_{N}\left(\begin{array}{ll}
\left\{\lambda_{p r}^{C}\right\}_{n} ; & \left\{\lambda_{a b}^{C}\right\}_{N-n} \\
\left\{\lambda_{p r}^{B}\right\}_{n} ; & \left\{\lambda_{a b}^{B}\right\}_{N-n}
\end{array}\right) \\
\quad=\left(\prod_{p r a b} f\left(\lambda_{p r}^{C}, \lambda_{a b}^{C}\right) f\left(\lambda_{a b}^{B}, \lambda_{p r}^{B}\right)\right) \mathscr{R}_{N}\left(\begin{array}{ll}
\left\{\lambda_{p r}^{C}\right\}_{n} ; & \left\{\lambda_{a b}^{C}\right\}_{N-n} \\
\left\{\lambda_{p r}^{B}\right\}_{n} ; & \left\{\lambda_{a b}^{B}\right\}_{N-n}
\end{array}\right) .
\end{aligned}
$$

The only singularities of rational function $\mathscr{R}_{N}$ are first order poles at $\lambda_{p r, j}^{B}=\lambda_{p r, k}^{C}$ and $\lambda_{a b, j}^{B}=\lambda_{a b, k}^{C}$. It also decreases as $1 / \lambda$ in each $\lambda$ at other $\lambda$ 's fixed.

(v) Now the proof of (5.3) can be done by induction in $N$, assuming that it is valid for $N \leqq M$. The proof of its validity for $N=M+1$ may be done by comparison of the residues of both sides at $\lambda_{p r, j}^{B}=\lambda_{p r, k}^{C}$ and $\lambda_{a b, j}^{B}=\lambda_{a b, k}^{C}$ using (4.17), (5.5). The residues appear to be equal which is sufficient for the proof of (5.3).

Formula (5.3) shows that

$$
\sigma_{n}^{\alpha}\left(\left\{\lambda_{p r}^{C}\right\}_{n} ;\left\{\lambda_{p r}^{B}\right\}_{n}\right)=\mathscr{R}_{n}\left(\begin{array}{ll}
\left\{\lambda_{p r}^{C}\right\}_{n} ; & \{\varnothing\} \\
\left\{\lambda_{p r}^{B}\right\}_{n} ; & \{\varnothing\}
\end{array}\right)
$$

(i.e. $\left\{\lambda_{a b}^{C}\right\}=\left\{\lambda_{a b}^{B}\right\}=\varnothing$, where $\varnothing$ is an empty set). This is the best way to introduce the function $\sigma_{n}^{\alpha}$ and to prove all its properties.

\section{Appendix C}

Let us prove Eq. (7.7). Consider matrix element $\mathbb{M}_{N}^{\alpha}(4.1)$ in special case $\ell_{j}^{C}=\ell_{j}^{B}=\ell_{j}$ and $m_{j}^{C}=m_{j}^{B}=m_{j}$. It can be represented in the form:

$$
\begin{aligned}
\mathbb{M}_{N}^{\alpha}\left(\left\{\lambda^{C}\right\},\left\{\lambda^{B}\right\},\left\{\ell_{j}^{C}\right.\right. & \left.\left.=\ell_{j}\right\},\left\{\ell_{j}^{B}=\ell_{j}\right\},\left\{m_{j}^{C}=m_{j}\right\},\left\{m_{j}^{B}=m_{j}\right\}\right) \\
& =\sum_{\text {part }}\left(\prod_{p r} m_{(p r)}\right)\left(\prod_{p r} \ell_{(p r)}\right) \mathscr{K}(\text { part }) .
\end{aligned}
$$

Coefficient $\mathscr{K}$ here depends on a partition and it is a rational function of $\lambda_{j}^{c}$, $\lambda_{j}^{B}(j=1, \ldots, N)$. According to the definition (7.3)

$$
I_{N}=\lim _{\varepsilon \rightarrow 0} \mathbb{M}_{N}^{\alpha}\left(\left\{\lambda_{j}\right\},\left\{\lambda_{j}+\varepsilon\right\},\left\{\ell_{j}\right\},\left\{\ell_{j}\right\},\left\{m_{j}\right\},\left\{m_{j}\right\}\right) c^{-N}\left(\prod_{j \neq k} f_{j k}\right),
$$

where one has to put $m_{j}=\ell_{j}^{-1} \prod_{k \neq j}\left(f_{k j} / f_{j k}\right)$ due to (2.12). On the other hand, form 
factor (4.16) is expressed in terms of $\mathbb{M}_{N}^{\alpha}(4.1)$ as follows:

$$
F_{N}^{\alpha}=\mathbb{M}_{N}^{\alpha}\left(\left\{\lambda^{C}\right\},\left\{\lambda^{B}\right\},\left\{\ell^{C}\right\},\left\{\ell^{B}\right\},\left\{m^{C}\right\},\left\{m^{B}\right\}\right),
$$

where one has to put $m_{j}^{C}=\left(\ell_{j}^{C}\right)^{-1} \prod_{k \neq j}\left(f_{k j}^{C} / f_{j k}^{C}\right)$ and $m_{j}^{B}=\left(\ell_{j}^{B}\right)^{-1} \prod_{k \neq j}\left(f_{k j}^{B} / f_{j k}^{B}\right)$. The limit (7.7) is easily seen to lead exactly to expression (C.2).

\section{Appendix D}

Let us consider the two-site model in the trivial situation where

$$
T_{1}(\lambda)=\left(\begin{array}{ll}
1 & 0 \\
0 & 1
\end{array}\right)
$$

which corresponds to $\ell(\lambda)=1$. Such $T_{1}(\lambda)$ satisfies all the requirements to be a monodromy matrix. In this case $\mathbb{B}_{1}(\lambda)=0$, which means that $\mathbf{Q}_{1} \prod \mathbb{B}\left(\lambda_{j}\right)|0\rangle=0$, and hence $\left\langle\psi_{N}\left(\lambda_{1} \ldots \lambda_{N}\right)\left|\mathbf{Q}_{1}^{2}\right| \psi_{N}\left(\lambda_{1} \ldots \lambda_{N}\right)\right\rangle=0$ and $I_{N}=0$. In our case $\ell_{j}=1$, but all $\lambda_{j}$ are some values satisfying s.t.e. (2.12). Hence $I_{N}\left(\left\{\lambda_{j}\right\},\left\{\ell_{j}=1\right\}\right)=0$.

Acknowledgement. We thank L. D. Faddeev for discussions.

\section{References}

1. Faddeev, L. D.: Quantum completely integrable models of field theory. LOMI-preprint P-2-79, Leningrad, 1979 and Sov. Sci. Rev., Math. Phys. C1, 107-160 (1981)

2. Creamer, D. B., Thacker, H. B., Wilkinson, D. : Gelfand-Levitan method for operator fields. Phys. Rev. D21, 1523-1528 (1980)

3. Smirnov, F. A.: Gelfand-Levitan equations for quantum nonlinear Schrödinger equation with attraction. Dokl. AN SSSR 262, 78-83 (1982)

4. Korepin, V. E.: Claculation for norms of Bethe wave functions. Commun. Math. Phys. 86, 391-418 (1982)

5. Korepin, V. E. : Analysis of the bilinear relation of the six-vertex model. Dokl. AN SSSR 265, 13611364 (1982)

6. Berezin, F. A., Pokhil, C. P., Finkelberg, V. M.: The Schrödinger equation for the system of onedimensional particles with point interaction. Vest. Mosk. Gos. Univ. Ser. 1.1, 21-28 (1964)

7. McGuire, J. B.: Study of exactly soluble one-dimensional N-body problems. J. Math. Phys. 5, 622636 (1964)

8. Brézin, E., Zinn-Justin, J.: Un problème à N corps soluble, C.R. Acad. Sci.(Paris) 263, 670-673(1966)

9. Mathematical physics in one dimension, Lieb, E. H. Mattis, D. C. (eds.). New York, London: Academic Press 1966

10. Gaudin, M.: La fonction d'onde de Bethe pour les modèles exacts de la mécanique statistique. Paris, Commisariat à l'energie atomique 1983

11. Korepin, V. E.: Correlation functions of the one-dimensional Bose gas in the repulsive case. Commun. Math. Phys. 93,

12. Izergin, A. G., Korepin, V. E.: Lattice versions of quantum field theory models in two dimensions. Nucl. Phys. B205 [FS5], 401-413 (1982)

13. Izergin, A. G., Korepin, V. E.: L-operator generating all the monodromy matrices with the $R$-matrix of the XXX-model. Zapiski Nauchn. Seminarov LOMI 131, 80-87 (1983)

14. Izergin, A. G., Korepin, V. E.: Pauli principle for one-dimensional bosons and the algebraic Bethe Ansatz. Lett. Math. Phys. 6, 283-288 (1982)

15. Faddeev, L. D., Sklyanin, E. K.: Quantum mechanical approach to completely integrable systems in field theory. Dokl. Akad. Nauk SSSR 243, 1430-1433 (1978) 
16. Sklyanin, E. K.: The inverse scattering method and the quantum nonlinear Schrödinger equation. Dokl. Akad. Nauk SSSR 244, 1337-1341 (1978)

17. Izergin, A. G., Korepin V. E., Smirnov, F. A.: Trace identities for the quantum nonlinear Schrödinger equation. Teor. Mat. Fiz. 48, 319-323 (1981)

18. Faddeev, L. D., Takhtajan, L. A.: Quantum inverse scattering method and the XYZ Heisenberg model. Usp. Mat. Nauk. 34, 13-63 (1979)

Communicated by A. Jaffe

Received August 31, 1983; in revised form December 8, 1983 